\title{
WestVirginiaUniversity
}

THE RESEARCH REPOSITORY @ WVU

Graduate Theses, Dissertations, and Problem Reports

2013

\section{A Survey of Journalism Schools' Online Publication of Student Content}

Sarah E. Moore

Follow this and additional works at: https://researchrepository.wvu.edu/etd

\section{Recommended Citation}

Moore, Sarah E., "A Survey of Journalism Schools' Online Publication of Student Content" (2013).

Graduate Theses, Dissertations, and Problem Reports. 6251.

https://researchrepository.wvu.edu/etd/6251

This Thesis is protected by copyright and/or related rights. It has been brought to you by the The Research Repository @ WVU with permission from the rights-holder(s). You are free to use this Thesis in any way that is permitted by the copyright and related rights legislation that applies to your use. For other uses you must obtain permission from the rights-holder(s) directly, unless additional rights are indicated by a Creative Commons license in the record and/ or on the work itself. This Thesis has been accepted for inclusion in WVU Graduate Theses, Dissertations, and Problem Reports collection by an authorized administrator of The Research Repository @ WVU. For more information, please contact researchrepository@mail.wvu.edu. 


\title{
Evaluating the Efficacy of Various Modalities to Improve Arterial Stiffness
}

\section{Corey Moore}

Thesis submitted to the School of Medicine at West

Virginia University

In partial fulfillment of the requirements for the degree of:

Master of Science in Exercise Physiology

\author{
Paul D. Chantler, Ph.D., Committee Chair \\ Randall W. Bryner, Ed.D. \\ I. Mark Olfert, Ph.D. \\ Melissa D. Olfert, Dr.P.H.
}

Department of Exercise Physiology

Morgantown, WV 2016

Keywords: Cardiovascular disease, arterial stiffness, metabolic syndrome, pulse wave velocity, aquatic exercise

Copyright 2016 Corey Moore 


\section{Abstract \\ Evaluating the Efficacy of Different Modalities to Improve Arterial Stiffness Corey Moore}

Metabolic syndrome (MetS) is associated with an increase in cardiovascular (CV) mortality, partially due to arterial stiffening, which can be measured non-invasively via pulse wave velocity (PWV). Arterial stiffness is a clinically relevant CV marker of mortality, an increase in PWV by only 1 SD is associated with an increased CV risk of 15\%. Land based aerobic exercise, both chronic and acute, has previously been identified as an effective method for improving arterial health. Unfortunately, land based aerobic exercise is not feasible for all populations, therefore a more effective treatment modality must be elucidated. Aquatic exercise has previously been shown to be beneficial for reducing blood pressure, as well as PWV. Fruit and vegetable (F\&V) consumption has been shown to decrease CV mortality, although the mechanism of action has yet to be fully elucidated. We tested the following hypotheses: 1) 8 week of dietary intervention, with increased fruit and vegetable consumption, as well as decreased complex carbohydrate and decreased fat consumption, will improve arterial hemodynamics 2) 8 weeks of aquatic exercise intervention will improve the PWV in women with MetS. 


\section{Acknowledgements:}

My time working on my Master of Science in Exercise Physiology has been trying and mentally stimulating time. There were times when I was pushed to my wits end, but I knew that I needed to persevere. My passion for learning, education, and medicine were some of my major motivators to push through, but that doesn't even come close to the support that I have received from my friends, family, and mentors. All of this support has pushed me to be the best that I can be and do everything to the best of my capabilities.

First and foremost, I would like to sincerely express my gratitude to my advisor Dr. Paul Chantler. He pushed me to be my best with a unique amalgam of encouragement, thought provoking questions, and strange British phrases, most of which I still I am still unsure the meaning. I truly appreciate your patience with me as I developed my skills, knowledge, and confidence over the past 2 years.

I would also like to extend my gratitude to the remainder of my committee. Dr. Bryner was one of the first professors to challenge me and push me to be the best I could be. He truly made me "thirst for knowledge", but I am not sure how many times I have been called a "goomba". Dr. Mark Olfert has pushed me to learn more about the pulmonary system and the adaptations during exercise than I ever could have imagined, but it has only made me a better person and scientist. I never had the pleasure of having Dr. Melissa Olfert in the classroom, but I did interact closely with her on my thesis project and she was always happy to help teach me nutritional concepts. I appreciate all of the time that each one of you have invested in me and helping to complete my thesis.

Friendship is one of the strongest bonds and can truly help people get through the most challenging moments in their life. I want to thank Erica Casto and Chris Skinner for being able to tolerate my non-sense at all times of the day, freaking out about whatever upcoming exam, quiz, or paper I had due. You really helped me to keep my head straight and give me the drive to finish. I would also like to acknowledge my roommate Hannah for consuming the innumerable baked goods I made while "procrasti-baking". I would like to thank everyone in the Chantler Lab, Evan DeVallance, Kayla Branyan, Sara Fournier, Shinichi Asano, and Kent Lemaster. I would also like to thank a few of the special interns who helped me with my project Kelly Devlin and Ashlee Seldomridge.

I would also like acknowledge my family and my wonderful fiancé. My love for science dates back to my early school days when my mother and father assured me that a career in the health science field would be the best for me. I listened to their advice and I would never look back. They have given me an immeasurable amount of support and encouragement throughout my education and for that I am eternally grateful. Finally, my fiancé, Kierra. She was the person who received the brunt of my stress and was forced to tolerate me through the worst of times. She was always able to keep me calm, cool, and collected, while pushing me to finish. I would not have been able to finish without all the love and support that you gave. 


\section{Table of Contents}

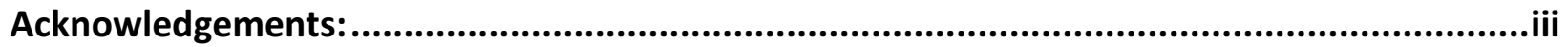

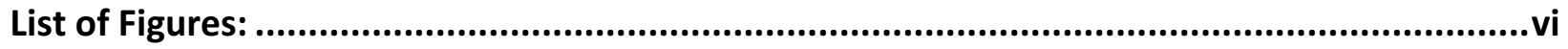

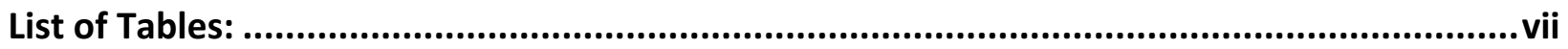

Abbreviations:............................................................................................................................... viii

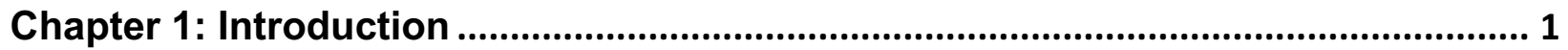

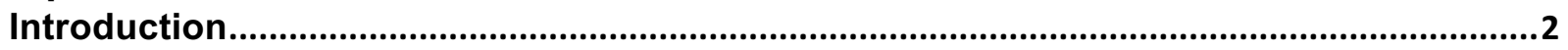

Specific Aims:......................................................................................................................

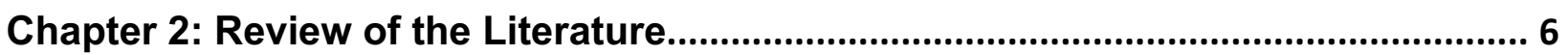

Prevalence of CVD and Obesity..........................................................................................

Cardiovascular Changes in a Younger Population .................................................................

Metabolic Syndrome ..............................................................................................................

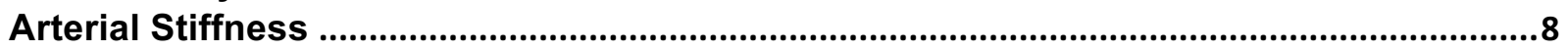

Dietary Intervention and Effects on Arterial Remodeling ...................................................11

Dietary Intervention and Arterial Stiffness..........................................................................13

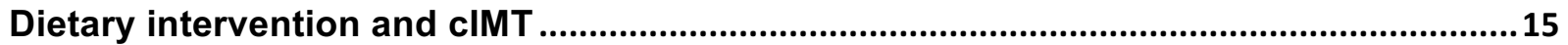

Aquatic Exercise Intervention ...........................................................................................17

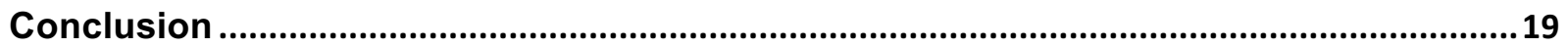

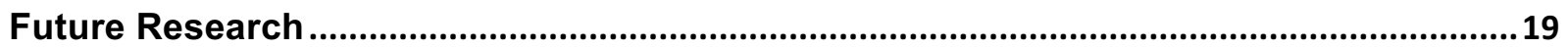

Chapter 3: Fruved Dietary Intervention Does Not Reduced PWV in Young-Adults.

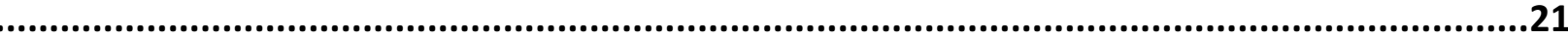

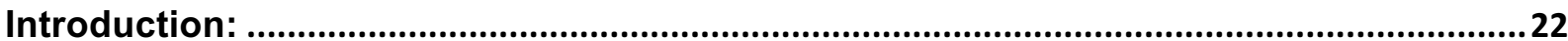

Methods:

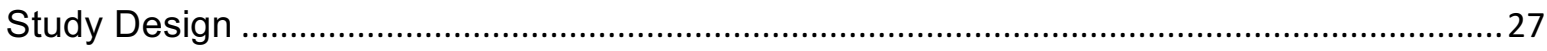

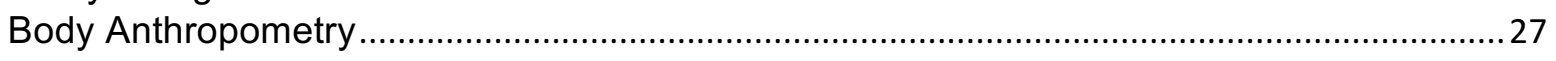

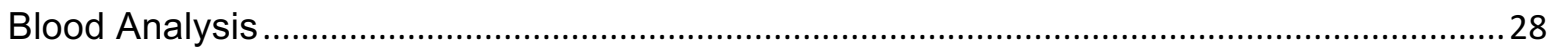

Arterial Geometry

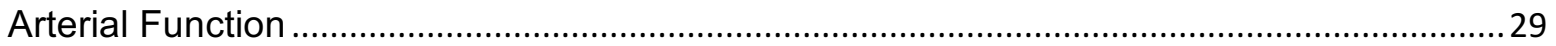

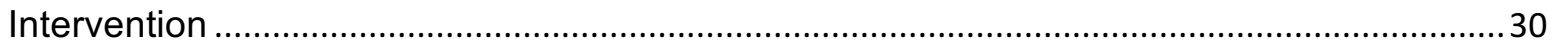

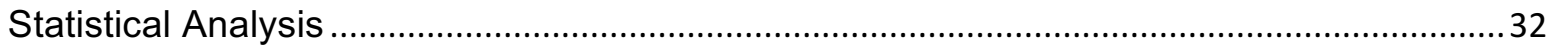

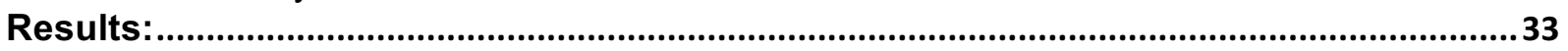

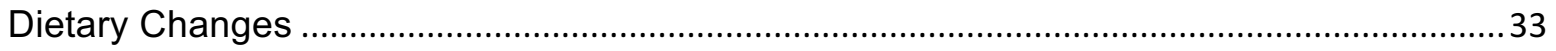

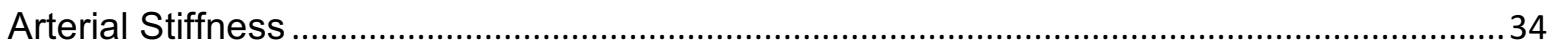

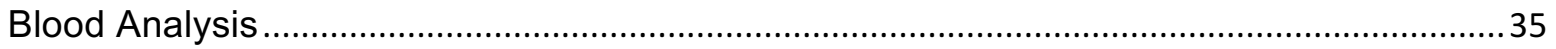

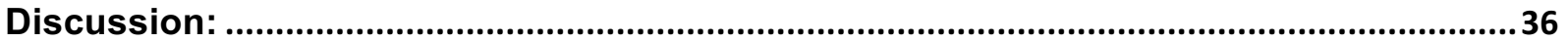

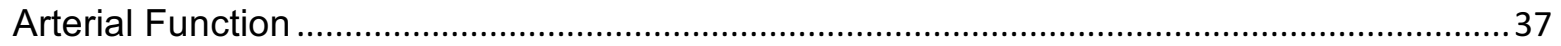

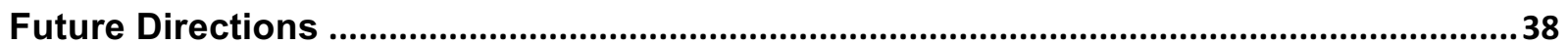

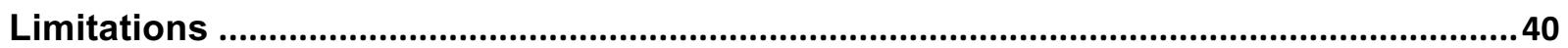

Chapter 4: Is Deep Water Aquatic Exercise an Effective Exercise Modality to Improve Arterial Stiffness in Women with Metabolic Syndrome? A Pilot Study ....45

Introduction: ..............................................................................................................................46

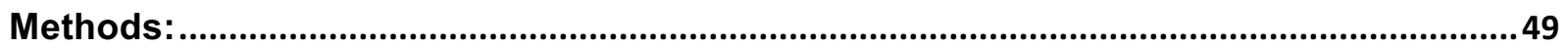




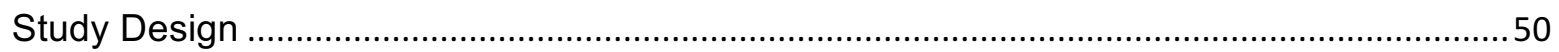

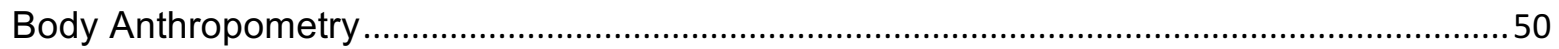

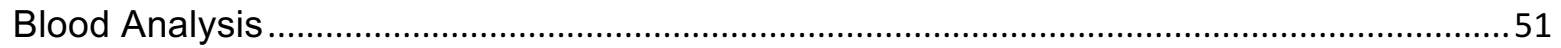

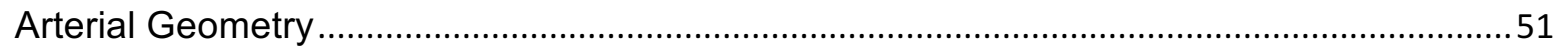

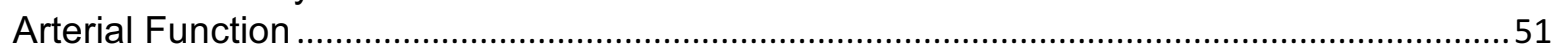

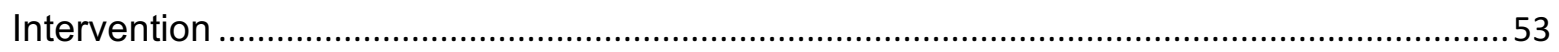

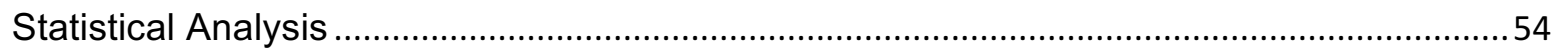

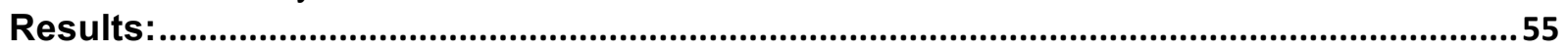

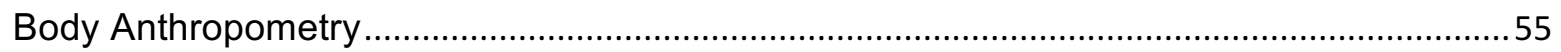

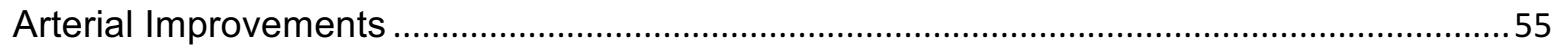

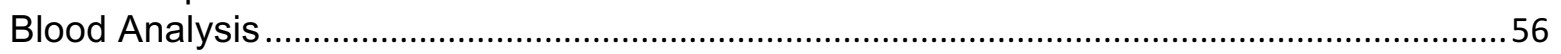

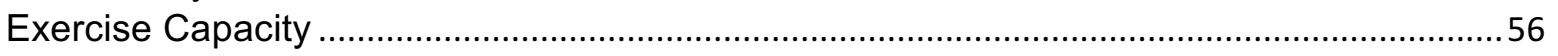

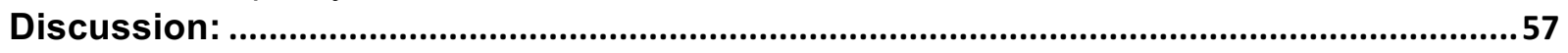

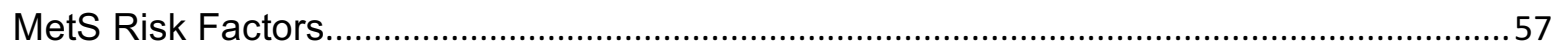

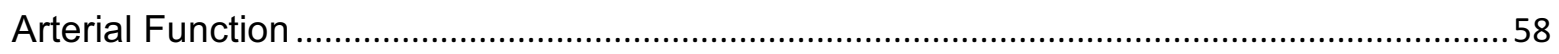

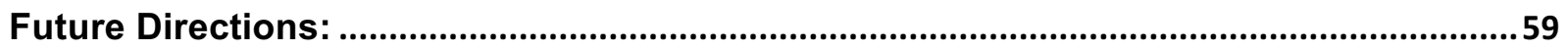

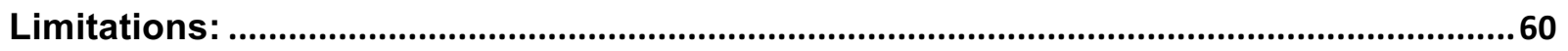

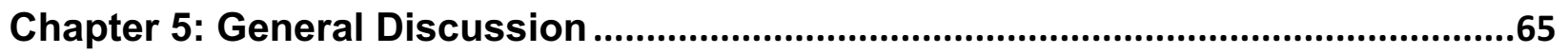

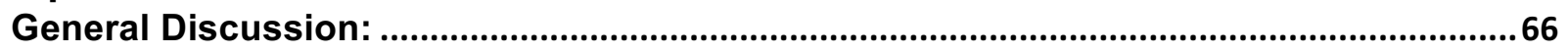

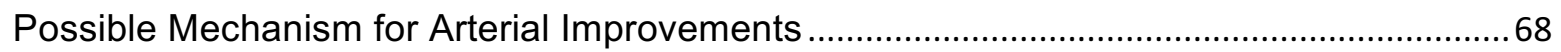

Future Directions: ................................................................................................................ 71

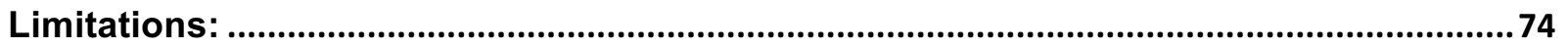




\section{List of Figures:}

Figure 1.1: Illustration of healthy arteries compared to stiffened arteries.......................... 1

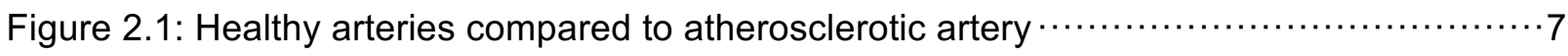

Figure 3.1: Changes in empty calories following 8 weeks of dietary intervention $\cdots \cdots \ldots \ldots \ldots \ldots . \cdots \cdots$

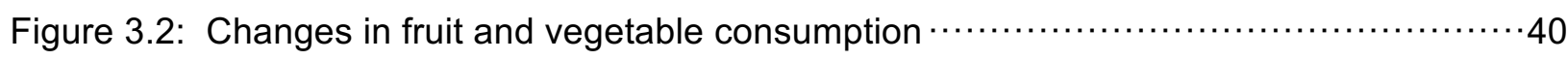

Figure 3.3: Changes in the Buckberg SEVR following FRUVEDomic dietary intervention $\cdots \cdots \cdot \cdots$

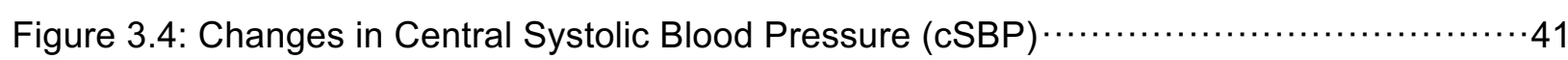

Figure 3.5: Changes in Carotid to Femoral PWV following 8 weeks of FRUVEDomic Dietary

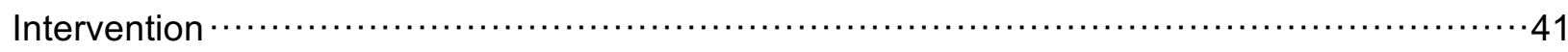

Figure 4.1: Changes in Body Mass Index (BMI) in response to 8 weeks of aquatic exercise

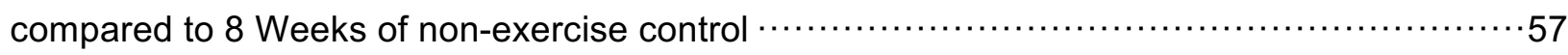

Figure 4.2: Changes in Pulse Wave Velocity (PWV) in response to 8 weeks of aquatic exercise

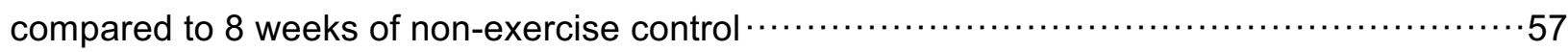

Figure 4.3: Changes in Augmentation Index (AGI) in response to 8 weeks of aquatic exercise

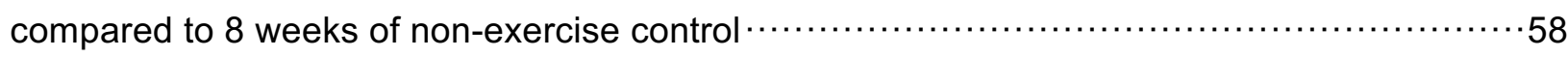

Figure 4.4: Changes in AGI, which has been adjusted to standardized heartrate (AGIHR) following 8 weeks of aquatic exercise compared to 8 weeks of non-exercise control 


\section{List of Tables:}

Table 3.1: Clinical characteristics of the FRUVEDomics cohort.

Table 3.2: Changes in arterial parameters following FRUVEDomics dietary intervention ......... 38

Table 3.3: Changes in blood characteristics following FRUVEDomics dietary intervention ...... 39

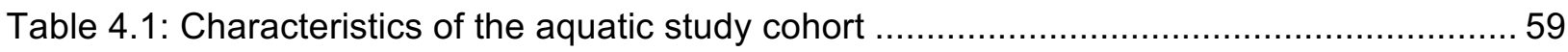

Table 4.2: Changes in arterial parameters following 8 weeks of aquatic exercise .................. 59

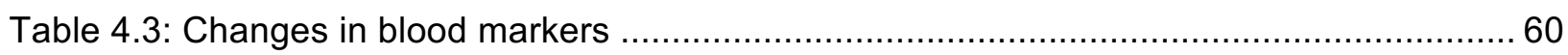

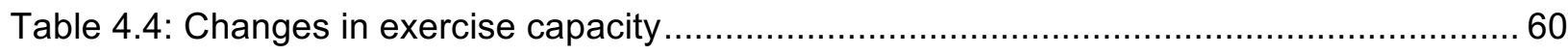




\section{Abbreviations:}

\begin{tabular}{|c|c|}
\hline AS & Arterial Stiffness \\
\hline $\mathrm{BP}$ & Blood Pressure \\
\hline cfPWV & Carotid to Femoral Pulse Wave Velocity \\
\hline CIMT & Carotid Intima Media Thickness \\
\hline $\mathrm{CV}$ & Cardiovascular \\
\hline CVD & Cardiovascular Disease \\
\hline $\mathrm{F} \& \mathrm{~V}$ & Fruit and Vegetable \\
\hline HDL & High Density Lipoprotein \\
\hline MetS & Metabolic Syndrome \\
\hline NCEP & National Cholesterol Education Program Adult Treatment Panel III \\
\hline NO & Nitric Oxide \\
\hline ROS & Reactive Oxygen Species \\
\hline MMP & Matrix Metalloproteinases \\
\hline TIMP & Tissue Inhibitor of Metalloproteinases \\
\hline eNOS & Endothelial Nitric Oxide Synthase \\
\hline PVAT & Perivascular Adipose Tissue \\
\hline IL & Interleukin \\
\hline TNF- $\alpha$ & Tissue Necrosis Factor alpha \\
\hline T2DM & Type 2 Diabetes Mellitus \\
\hline
\end{tabular}




\begin{tabular}{|c|c|}
\hline bSBP & Brachial Systolic Blood Pressure \\
\hline bDBP & Brachial Diastolic Blood Pressure \\
\hline cSBP & Central Systolic Blood Pressure \\
\hline cDBP & Central Diastolic Blood Pressure \\
\hline Alx & Augmentation Index \\
\hline PUFA & Poly Unsaturated Fatty Acid \\
\hline $\mathrm{CO}$ & Cardiac Output \\
\hline WV & West Virginia \\
\hline USDA & United States Department of Agriculture \\
\hline $\mathrm{AHA}$ & American Heart Association \\
\hline ED & Eating Disorder \\
\hline BMI & Body Mass Index \\
\hline BSA & Body Surface Area \\
\hline CCA & Common Carotid Artery \\
\hline PP & Pulse Pressure \\
\hline SEVR & Subendocardial Viability Ratio \\
\hline EKG & Electrocardiogram \\
\hline $\mathrm{RD}$ & Registered Dietician \\
\hline ASA-24 & Automated Self-Administered 24 Diet Recall \\
\hline ANOVA & Analysis of Variance \\
\hline TKcal & Total kilocalories \\
\hline $\mathrm{CHO}$ & Carbohydrate \\
\hline
\end{tabular}




\begin{tabular}{|c|c|}
\hline HTN & Hypertension \\
\hline bMAP & Brachial Mean Arterial Pressure \\
\hline LDL & Low Density Lipoprotein \\
\hline AGE & Advanced Glycation End-Products \\
\hline BPM & Beats Per Minute \\
\hline HR & Heartrate \\
\hline AGI & Augmentation Index \\
\hline AGIHR & Augmentation Index Standardized to HR \\
\hline HRR & Heartrate Reserve \\
\hline WC & Waist Circumference \\
\hline $\mathrm{HC}$ & Hip Circumference \\
\hline $\mathrm{BF} \%$ & Body Fat Percentage \\
\hline VE & Ventiliatory Equivalent \\
\hline VT & Ventilitory Threshold \\
\hline HRMAX & Heartrate Maximum \\
\hline RER & Respiratory Exchange Ratio \\
\hline VO2 & Volume of Oxygen Consumed \\
\hline CRP & C-Reactive Protein \\
\hline $\mathrm{HbA1c}$ & Glycated Hemoglobin \\
\hline SNS & Sympathetic Nervous System \\
\hline
\end{tabular}




\section{Chapter 1: Introduction}


Introduction

Arterial stiffness (AS) is characterized by the loss of elasticity of the arteries, and thus leads to an increase in the pressure within the vessels, and this increase has been shown to increase the risks of stroke and other cardiovascular diseases [1].

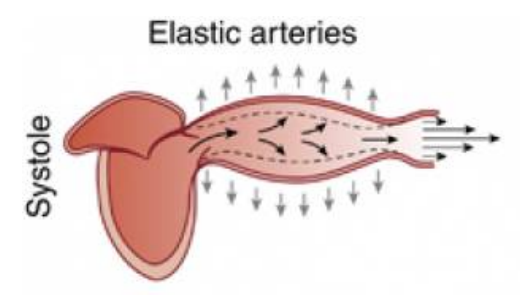

$\searrow$ Systolic/pulse pressure 入 Diastolic flow

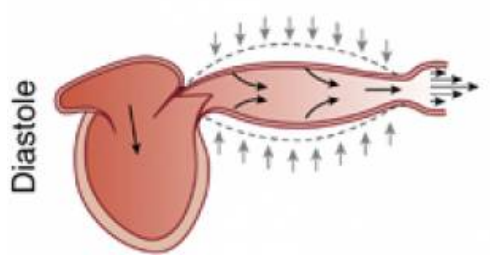

Stiff arteries

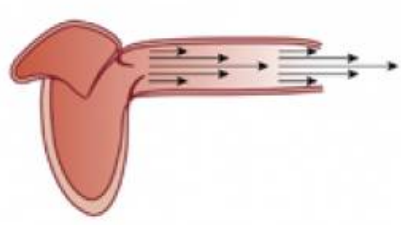

入 Systolic/pulse pressure $\checkmark$ Diastolic flow

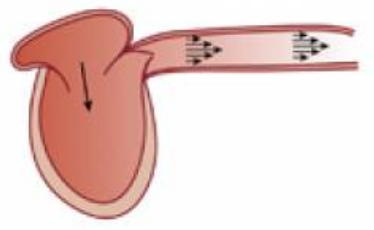

Figure 1.1: Illustration of healthy arteries compared to stiffened arteries in systole and diastole of the vessel

The arteries are known to stiffen naturally as humans age, but they also stiffen as a manifestation of many cardiovascular (CV) risk factors, such as obesity, hyperlipidemia, hypercholesterolemia, among other causes. Pulse wave velocity (PWV) is considered the gold standard method for quantifying AS, with a decrease in PWV of $1 \mathrm{~m} / \mathrm{s}$ corresponding to a $15 \%$ decrease in cardiovascular risk [1]. Exercise and nutritional intervention are two major mechanisms through which AS can be attenuated; both of these methods are effective at lowering the $\mathrm{CV}$ risks associated with AS, thus one or both of these methods can be used to lower AS [2].

AS can be partially attributed to arteriosclerosis, which is general and unspecified hardening, and loss of elasticity or medium and large arteries, which occurs with aging, or it can be attributed to atherosclerosis, which is arterial hardening specifically due to the 
deposition of atheromatous plaque [3]. Atherosclerotic disease is a natural occurrence with age, with the thickening of the arterial walls being one of the major implications. The arterial layer, where plaque builds, up is called the intima media, and therefore as this intima media thickens, the risk for cardiovascular disease (CVD) increases. The thickness of the intima media can be measured through Doppler ultrasound, and is typically performed in the carotid artery. An increase in carotid intima media thickness (cIMT), is indicative of CVD risk. A decrease in cIMT by $0.1 \mathrm{~mm}$ correlates to a $16 \%$ decrease in the risk for stroke or myocardial infarction [1].

Land based aerobic exercise has been shown to be effective at improving AS, however, it is not feasible for every population, particularly those at increased risk of fall or CV mortality [1]. Aquatic exercise has been shown to be effective at reducing blood pressure following 10 weeks of aquatic exercise in multiple studies [4, 5]. Shallow water exercise has previously been shown to reduce arterial stiffness, as measured by PWV, in older individuals following a 10 week exercise intervention [6]. Reductions in PWV are often attributed to decreases in $\mathrm{BP}$, however, this decrease in PWV was not accompanied with reductions in BP. Therefore, there must be a different mechanism at work, which must be elucidated. Although, many individuals believe that an exercise intervention is too intensive to incorporate into their lifestyle, thus the determination of how effective a nutritional intervention is at improving AS is of critical importance.

Perhaps, a less intensive lifestyle intervention could be to alter the diet to include a high proportion of fruits and vegetables. Fruits and vegetables have been reported to be cardio-protective, and have been suggested to exert reductive effect on AS [2]. Previous studies have presented inconclusive results of specific diet supplementation and 
their effects on AS, but few have looked at a major diet intervention across a population of varying health levels [2, 7-11]. Long term studies often associate diets high in fruits and vegetables with improved cardiovascular risk factors [12-14], however, studies investigating supplementation, with individual components or macromolecules have had inconclusive results [15]. Thus, the determination of the exact mechanism behind the improvements are not simply one component of the fruits or vegetables, rather a more complex synergeistic interaction between the components and the body. Fruits and vegetables have been shown to improve AS through increased bioavailability of NO, decreased cIMT, and improved endothelial function [16]

The significant impact that small changes in vascular measurements illustrates the critical need for a feasible method for individuals to improve AS. While aerobic exercise has been consistently shown to lead to improvements in vascular function, the number of individuals who follow an exercise program is approximately $32 \%$, with approximately $50 \%$ of individuals quitting an exercise routine in the first 6 months, citing the time commitment as the major reason for quitting $[1,2,10,11]$. Therefore, a feasible strategy could potentially be a nutritional intervention.

Fruits and vegetables have a plethora of benefits to the body, from being cardioprotective to improving gastrointestinal function [2]. It is also suggested that a diet high in fruits and vegetables would result in a decrease in AS $[2,7,10,11]$. When increasing the amount of fruits and vegetables in the diet it is likely that the amount of high fat foods in the diet will be reduced, which help to further bolster the effects of a fruits and vegetable diet. This would lead to decreased AS due to the decreased circulating lipids, which improves endothelial function and leads to decreased inflammatory activity $[17,18]$. 
There has been intense research, with regards to certain components in fruits and vegetables and their effects on vascular function, for example flavonoids, which are plant pigments, have been shown to improve microvascular reactivity in a dose-dependent manner $[2,10]$. Flavonoids have been shown to scavenge free radicals as well as prevent their formation, notably ROS [19]. These ROS have also been linked to endothelial impairment in the short term and endothelial dysfunction in the long term, this means that flavonoids could function through a mechanism that augments endothelial function, by reducing the number of ROS.

Specific Aims:

Aim 1: Utilize fruit and vegetable diet based intervention, with $50 \%$ of meals being fruits and vegetables, to assess changes in, and characterize cardiovascular assessments of function.

Aim 2: Determine if low carbohydrate or low fat diets in conjunction with the fruit and vegetable diet lead to further changes in cardiovascular function.

Aim 3: Evaluate a deep water aquatic exercise program and its efficacy at improving measurements of cardiovascular function.

We hypothesize that both the fruit and vegetable dietary intervention and the aquatic exercise intervention will both lead to improvements in AS, as measured by PWV. 
Chapter 2: Review of the

Literature 
Prevalence of CVD and Obesity

Cardiovascular disease (CVD) is the leading causes of death in the US, with mortality rates of 611,105 per year [20]. The number of individuals in the US that suffer from CVD is approximately 26.6 million, which correlates to $11.3 \%$ of the population [20]. These mortality rates result in a healthcare cost burden of $\$ 147$ billion [21]. One of the major risks factors that is associated with the development of CVD is excess adiposity. The obesity rates in the US have been rapidly rising, with $34.9 \%$ of the adult population being categorized as obese in 2011-2012 [22].

Cardiovascular Changes in a Younger Population

Also rising at an alarming rate, is the prevalence of obesity in the younger populations, with $16.9 \%$ of children between the ages $2-19$ being categorized as obese [22]. Astoundingly, children are also being diagnosed with diseases that were previously only thought to occur in adulthood, such as type 2 diabetes. Le et al. found that $75 \%$ of obese children, caused by energy imbalcnce, such as that caused by sedendary lifestyle and increased empty calories, with a mean age of approximately 13 , had an advanced arterial age [23]. One of the major implications of long-term obesity is the development of the metabolic syndrome (MetS) which is a clustering of metabolic risk factors within a given individual, therefore the prevalence of MetS is also steadily increasing.

\section{Metabolic Syndrome}

Metabolic syndrome is characterized by a cluster of malignancies, which lead to an increased risk of cardiovascular related mortality. MetS was first described in 1988 as 
"syndrome X" when describing a cluster of co-morbidities, which lead to insulin resistance. These co-morbidities were central obesity, hypertension, and diabetes [24]. This cluster of co-morbidity is now recognized as the MetS. The guideline that indicate the comorbidities are detailed by the National Cholesterol Education Program Adult Treatment Panel III (NCEP), which requires that at least three of five of the guidelines be met. These guidelines are abdominal obesity, hypertension, hyperglycemia, hypertriglyceridemia, and decreased high density lipoprotein (HDL) [25].

MetS leads to an increased risk for CVD, when compared to healthy individuals. The presence of MetS is associated with a 3-fold increase in the risk for a cardiovascular incident, including myocardial infarction and cerebrovascular accident [1]. There is conflicting evidence regarding the underlying mechanism for this increased risk [26]. Studies have shown that the development of MetS is associated with an increase in the stiffness of the large conduit arteries, such as the aorta [26-28]. Other studies have shown that the development of MetS is associated with endothelial dysfunction [26, 28].

\section{Arterial Stiffness}

AS, which is a natural occurrence as we age, is characterized by loss of compliance of blood vessels and leads to an increase the risk for cardiovascular incidence. There are methods, such as exercise, that can be utilized to mitigate the extent to which the vessels stiffen and to offset non-age related stiffening of vessels $[1,26]$.There are many risk factors, aside from age, that can lead to the alterations in AS, and these include obesity, hypertension, hyperlipidemia, hypercholesterolemia, and high fasting blood glucose levels. 
The arteries are the vessels through which blood is transported to the periphery of the body. These vessels are composed of three layers, the tunica intima, the tunica media, and the tunica adventitia (Figure 1). The composition of these layers play a major role in the stiffness of vessels, and can change given different conditions.

The Structure of an Artery Wall

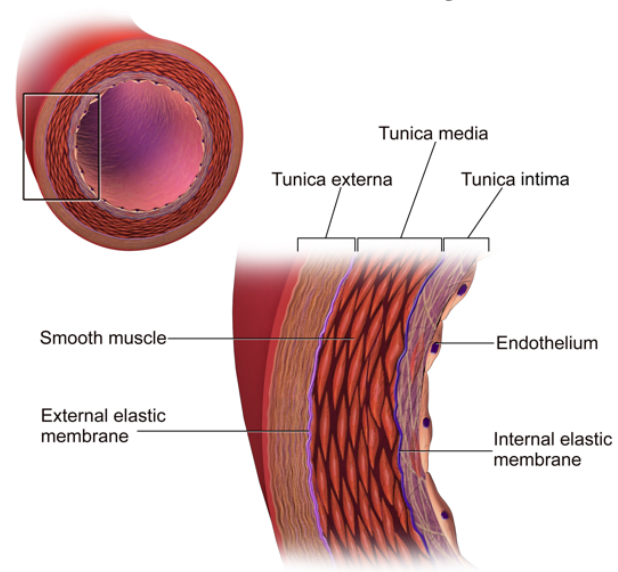

Atherosclerosis

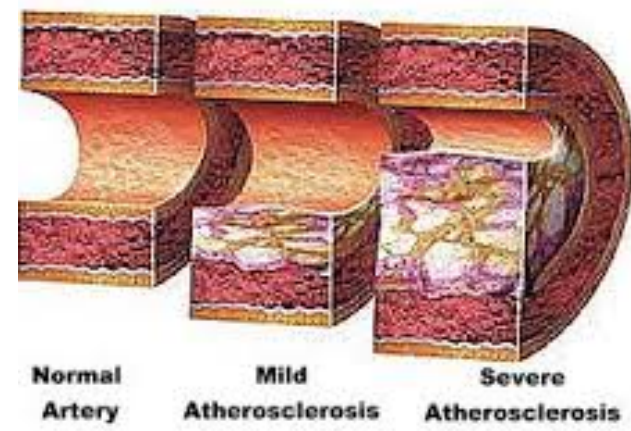

Figure 2.1: The structural components of the layers of a healthy artery, when compared to the structural changes with atherosclerosis

Modulation of AS occurs through multiple mechanisms, which involve structural or functional changes to the artery. Structural changes are often associated with changes in the levels of elastin and collagen, while functional changes are related to changes in the function of the endothelial, particularly in response to stress.

Elastin and collagen are the two major structural proteins, which modulate AS based on the amount of each in the extracellular matrix. Elastin is the component that lends greater ability of compliance to the vessels. Collagen lends a greater rigidity to the vessels. Therefore, with an elevated collagen leads to a stiffer and less compliant vessel [28].

Matrix metallopeptidase-9 (MMP-9) and tissue inhibitor of metallopeptidase-1 (TIMP-1) have been shown to be elevated in hypertensive subjects, when compared to 
healthy controls [27]. MMP-9 has been shown to break down elastin and collagen in the extracellular matrix [27]. Collagen is degraded very slowly, and are often cross-linked with other partially degraded collagen fibers, through enzymatic or non-enzymatic glycation. The products of this cross-linking are typically present in a less organized fashion and lead to a further stiffening of the vessel [26].

Changes in the functional aspect of the artery that augment stiffness are often associated with nitric oxide bioavailability. Nitric oxide is a potent vasodilator of the arterial endothelium; it is the primary modulator of arterial tone in response to shear stress. As the heart contracts and blood is propagated throughout the arterial system, there is a force that is exerted on the arterial wall, this force is called shear stress. The force activates mechanoreceptors that are located within the vascular smooth muscles cells. Through this mechanism, if a large enough amount of shear stress is detected, then eNOS is activated. This eNOS is responsible for synthesizing NO, which is used to modulate the tone of the artery, to prevent damage [29]. eNOS produces NO, by converting arginine to citrulline, generating $\mathrm{NO}$ as a by-product of this reaction [30].

Endothelial dysfunction occurs when the endothelium is not able to respond properly to the shear stress by dilating. Often, this dysfunction is associated with decreased NO bioavailability, either through decreased NO production or through increased NO consumption by ROS [30]. This decreased ability to augment the diameter of the artery, leads to an increased pressure in the vessel and can lead to damage the walls of the artery. This functional damage can lead to structural damage, which means the structural and functional changes in AS are inter-related and can lead to alterations in one another [29]. 
Two of the major strategies to lower these cardiovascular risk factors and PWV are nutritional intervention and the introduction of an exercise program $[1,12]$. Due to the profound affect that even minor changes in AS can have on the vascular function, it is an important area of research to investigate [1].

\section{Dietary Intervention and Effects on Arterial Remodeling}

A double-edged sword in the development of CVD is diet, because diet often is a risk factor for the development of CVD, but can also be used to combat CVD. Diets high in sodium, saturated fat, trans fat, and glucose have all been implicated in the development of MetS [31-33]. Given the relationship between hypertension and AS, the suggestion that diets, which can potentially exacerbate hypertension, also lead to the development of AS. Diets that are high in sodium, which have been defined as greater than $2,400 \mathrm{mg}$ of sodium consumption per day, have long been known to have some effect in the development of hypertension, and sodium has also been shown to have some effect in the development of AS [34,35]. Elevated levels of sodium has been shown to damage glycocalyx, which is a lining on the outer portion of blood vessels [34]. Damaging the glycocalyx results in changes in the permeability of vessels and it may also augment the release of nitric oxide, a major modulator of arterial tone [35].

Consumption of a diet high in saturated and trans fat also has many implications in the progression of AS. Diets high in fats are implicated in the production of perivascular adipose tissue (PVAT), which acts in a paracrine fashion releasing hormones, cytokines, interleukin-6 (IL6), and TNF- $\alpha$ [36]. Some cytokines, such as IL-6 and TNF- $\alpha$, act in an inflammatory manner, and therefore an increase will lead to greater systemic 
inflammation. This inflammation can cause damage to the arterial walls, leading to a breakdown of collagen molecules. These partially degraded collagen molecules can be cross-linked through glycation, leading to stiffer arteries [32]. In addition to fats, other dietary factors may have an influence on AS, such as diets high in simple $\mathrm{CHO}$ or refined sugars.

Excessive consumption of glucose-rich diets have implications in the development of type 2 diabetes mellitus (T2DM), which has its own host of CV implications, but it may also play a role in the development of AS, which often precedes T2DM [31]. Diets high in simple carbohydrates have been correlated to an increase in systolic blood pressure (SBP) and also increased PWV. These diets are also associated with an increase in blood triglyceride levels and reduced high density lipoprotein levels. Mechanistically, the implication of changes in arterial compliance are mediated through the increase in the SBP [26]. These elevations in SBP and PWV that result from glucose rich diets have been postulated to be the outcome from increased ROS production, increased expression of adhesion molecules, and increased advanced glycation end products [37]. Utilizing various dietary interventions as a method of improving arterial health is a sprawling area of research. Many dietary interventions exist that attempt to improve arterial health, in terms of decreasing arterial stiffness. These dietary interventions can be short-term dietary supplementation $[15,38-41]$ or they can investigate the lifelong diet and assess those that ate greater portions of fruits and vegetables [12] and assess changes. 


\section{Dietary Intervention and Arterial Stiffness}

Various studies [15, 38-41] have investigated changes in AS, as measured by PWV, in individuals of varying health and age, with different short-term dietary interventions. These studies investigated the efficacy of short-term diets (between 1 single dose and 14 weeks of intervention) and determined how the augmentation of the diet affected AS in their cohorts. There were conflicting results that were achieved between the various experimental designs [15, 38-42].

Bondonno et al. [38] used a dietary intervention, in which there were two diet periods, 7 days each. The first diet was a low nitrate diet, in which the participants were required to consume less than $100 \mathrm{mg} /$ day of nitrates. Nitrates are a necessary component of the diet, because they can be reduced to nitric oxide, a potent vasodilator, which help to regulate blood pressure, among other processes [28]. The second diet consisted of a diet high in nitrates, where the participants were advised to increase the consumption of beets and leafy green vegetables, in which the participants were required to increase their baseline consumption of nitrates by $300 \mathrm{mg} / \mathrm{d}$. The major findings of this study reported no significant changes in PWV, with the low nitrate group values of PWV: pre of $7.8 \mathrm{~m} / \mathrm{s}$ and post of $7.7 \mathrm{~m} / \mathrm{s}$ and the high nitrate group values of PWV: pre of 8.0 $\mathrm{m} / \mathrm{s}$ and post of $7.9 \mathrm{~m} / \mathrm{s}$. In another study investigating dietary intervention on PWV Lynn et al. [39] investigated the use of a pomegranate juice, which was chosen because it has been suggested to be an anti-atherosclerotic. The study consisted of 2 groups. One group consumed $330 \mathrm{ml} / \mathrm{d}$ of pomegranate juice, while the other group consumed an equivalent portion of lemon soda as a control drink. This intervention duration was 4 weeks, and had 51 participants. The major findings, showed no changes between the groups in PWV 
$(8.03 \mathrm{~m} / \mathrm{s}$ vs $8.28 \mathrm{~m} / \mathrm{s}, \mathrm{p}=0.694)$. Both of these studies had a similar demographic in terms of age (middle aged adults), health level (moderately healthy), and gender spread (approximately 2:1 female to male).

In contrast to the above, dietary interventions have been shown to lower PWV. McAnulty et al. [40] used an approach in which the typical diet of the participants was supplemented with blueberry powder. This was selected in an effort to increase the flavonoids and proanthocyanidins in the diet, which are speculated to reduce blood pressure. One group ingested blueberry powder (BB) that was equivalent to $250 \mathrm{mg}$ of whole blueberries per dose. The second group was provided an equivalent placebo powder (PP). The supplementation took place over the course of 6 weeks, in which the respective powders were ingested 1 dose per day. Major findings were that the BB group had a significant decrease in augmentation index (Alx, an alternative measure of arterial stiffness, 18.91 to 14.66 with $p=0.011$ ) and brachial systolic blood pressure (bSBP, 117.23 to 114.15 , with $p=0.047$ ), while there were no changes in the PP group. These findings were corroborated by Monahan et al., [15] who investigated the changes in central and peripheral blood pressure, as well as PWV with supplementation of diet with omega-3 polyunsaturated fatty acids (PUFA) for 12 weeks. The rationale for utilizing PUFA was that they have been shown to have a reductive effect on blood pressure and they have been shown to reduce inflammation. They showed that supplementation with PUFA decreased PWV $(9.88 \mathrm{~m} / \mathrm{s}$ to $8.95, \mathrm{P}<0.05)$ in older individuals. Kim et al. [41] indicates that PWV can be decreased through the use of nitrate supplementation in young healthy men. The participants in this study were given $140 \mathrm{~mL}$ of beet root juice (BR) or $140 \mathrm{~mL}$ of nitrate depleted equivalent (ND) in 1 acute dose. Results showed that the BR 
group had a significant decrease in PWV compared the to the ND group 3 hours following ingestion.

While investigating the changes associated with a short-term change in the diet is useful for determining what interventions can be useful in the amelioration of $A S$, it is also important to investigate how chronic diets change AS. Aatola et al. [12] investigated the changes in PWV associated with lifelong increase in fruit and vegetable consumption. This study examined a group of individuals over the course of 27 years and analyzed their diet. At the end of the 27 year follow up, the participant's PWV was evaluated. The major findings were that the individuals that were categorized as consuming high amounts of fruits had a lower PWV than their peers that reports low amounts of fruits $(P=0.03)$. It was also determined that those that consumed high amounts of vegetables had a lower PWV compared to those that consumed low amounts of vegetables $(P=0.03)$.

\section{Dietary intervention and cIMT}

As humans age there is a natural occurrence and progression of atherosclerotic disease, which to some extent this is unpreventable. However, the extent to which this accumulation occurs is modifiable, based on many factors such as diet and exercise [43, 44]. The layer in the vessels that sees this accumulation is called the intima media layer, and the thickness of this layer is typically measured via ultrasound. The most common vessel that is measured is the carotid artery with this measurement being called the carotid intima media thickness (cIMT) [44]. There are many factors that can slow the progression or potentially even reverse cIMT, such as physical activity and diet [45]. There have been many studies that have investigated the role that diet can play in 
preventing or slowing the increase in cIMT. Many studies have investigated dietary interventions and their effects on the progression of cIMT [13, 14, 43]. These studies investigated lifelong diet and assessed how it changed the cIMT or a long-term dietary intervention and assessed how cIMT changed.

McClintock et al. [43] collected the food consumption records of a group of participants and analyzed them for various components. A follow-up assessment was performed on the same participants 10 years later to determine changes in the diet and at this second visit cIMT measurements were performed. Results showed that those that ate a more balanced diet, consisting of rice, red meat, fish, fruits, and vegetables had a slower progression of cIMT than those that consumed a diet based in animal protein, gourds, and roots. Overall, there was an inverse association between fruits and vegetables and cIMT progression. Ellingsen et al. [14] found similar results where a group of individual's diets were assessed for levels of fruit and berry consumption. The authors report that there is a negative association between the consumption of fruits and berries and progression of cIMT levels. Furthermore, individuals that showed the largest consumption of berries and fruits also had the slowest progression of cIMT levels.

Petersen et al. [45] and Ellingsen et al. [13] both investigated dietary interventions of increasing fruits and vegetables and vitamin C consumptions respectively, and progression of cIMT in elderly men at risk for CVD. Both of these studies found that there was an association with the dietary intervention and progression of cIMT. Petersen et al. showed that, following a 12-month dietary intervention of increased fruits and vegetables; there was a slowed progression of cIMT levels compared to non-intervention control group. Ellingsen et al. [13] found that following a 3 year intervention of increasing vitamin 
C consumption, the cIMT of the dietary intervention group had a slower progression compared to the non-intervention control group.

Based on these study results fruit and vegetables appear to have a major impact on the progression of cIMT. cIMT progression occurs naturally as humans age, but it appears to be attenuated with increased fruit and vegetable consumption. Given the implications of cIMT in the development of CVD, increased consumption of fruits and vegetables has many implications in preventing or slowing the development of CVD.

\section{Aquatic Exercise Intervention}

While land based aerobic exercise has previously been shown to be an effective method for attenuating increased AS; land based exercise is not always an appropriate mode of exercise. Individuals with injuries such as osteoarthritis, balance abnormalities, or other injuries may not able or may not be willing to participate in the higher impact landbased exercise. These individuals have previously forgone exercise and were not able to glean the major benefits associated, but aquatic exercise has shown promise in improving $\mathrm{CV}$ risk factors. While there is limited literature available on exercising in an aquatic environment, the research that has been performed is promising.

Simply being in an aquatic environment leads to many alterations to the baseline metabolic functions of the cardiovascular system to compensate for the environmental changes. Immediately upon submersion in water, a hydrostatic pressure will be exerted onto the entire submersed portion of the body. This pressure will lead to many cardiovascular changes, such as an increase in stroke volume, which may be associated with an increase in central venous pressure or decreased vascular resistance, and a 
decrease in heart rate [46]. These changes all lead to an increase in the total cardiac blood volume. These changes alone, lead to complete overhaul in the exercise dynamics when compared to land [46].

Aquatic exercise has been shown to have many beneficial effects on the body physiology and in theory should be an effective method to mitigate the negative cardiovascular effects of obesity and MetS. Upon being placed in the water, the heart has an increased stroke volume, due to increased venous return. Given this increased stroke volume, there is a proportional decrease in $\mathrm{HR}$, which is necessary to maintain cardiac output (CO) [46].

Many studies $[6,47,48]$ have investigated the effects of aquatic exercise on the arterial function of a variety of populations, and have shown an improvement in their measures of arterial function [6]. Ten weeks of shallow water aerobics has been shown effective at decreasing the PWV of sedentary adults [6]. Similarly, Nualnim et al. investigated 12 weeks of swimming exercise in pre-hypertensive or stage-1 hypertensive individuals. They found that the introduction of the swimming exercise lead to a $21 \%$ increase in arterial compliance in the carotid, as well as significant improvements in flow mediated dilation and baroreflex sensitivity. Additionally, Alkatan et al. investigated swimming based exercise and its effect on PWV compared to land based exercise [49]. The major findings of this study illustrate that PWV is reduced comparably in the swimming group, when compared to the land based aerobic exercise. Lee et al. utilized a different population, obese elementary students, to determine if these benefits translated to a younger population [48]. They report that the arterial compliance in the lower extremity improved significantly in the swimming group, compared to the control 
group [48]. PWV reductions are often accompanied with a reduction in central and peripheral blood pressure, therefore a reduction in central blood pressure may precede changes in AS. Multiple studies $[5,50,51]$ have all shown that that aquatic based exercise is as effective as land based exercise at reducing blood pressure.

\section{Conclusion}

Fruit and vegetable consumption has been reported as heart-healthy, but there has been conflicting data on the mechanism of action. Fruits and vegetable consumption appear to play a major role in the prevention of AS. Although, in studies of increased fruit and vegetable consumption or consumption of nutrients from fruits and vegetable, there were some conflicting findings. Diets with a shorter invention, 4 weeks or less, reported no changes in PWV $[38,39]$. However, these interventions may not allow adequate time for arterial remodeling, a major mechanism in decreasing AS, to occur. Diets with a more long-term invention $[15,40]$ (6-12 weeks) as well as lifelong interventions [12] showed decreased PWV, which is indicative of decreased AS. cIMT was another factor in AS that has been implicated in CVD. Studies have reports that increased consumption of fruits and vegetables slowed progression of the cIMT. In conclusion, the consumption of fruits and vegetables, in the long-term, appear to be effective at reducing AS and slowing the natural progression of cIMT.

\section{Future Research}

Given the current state of the literature, there are a number of gaps in the research. Many interventions require the participants to consume a supplementation, which could 
be expensive or simply not feasible in everyday life. Therefore, it is necessary to develop an intervention that can be non-invasively integrated into an individual's lifestyle. There is a clear age gap in the research, with the majority of the focus being placed on either a pediatric population or or an adult population. There is a huge lack of research in a college aged, young adult population. There were very few studies investigating changes blood biomarkers of arterial remodeling, MMP-9, TIMP-1, IL-6, and TNF- $\alpha$, these studies should be further investigated to elucidate, to determine the mechanism of action. 


\section{Chapter 3: Fruved Dietary}

Intervention Does Not

Reduced PWV in Young-

Adults. 
Introduction:

Cardiovascular disease (CVD) is the leading cause of death in the US with approximately 611,000 deaths attributed annually, and a total of 26.6 million individuals in the US diagnosed [20]. CVD is also associated with a colossal health care cost burden, totaling over $\$ 147$ billion [21]. Obesity is one of the major factors in the development of CVD, it has been a growing problem in the United States with $34.9 \%$ of the population being categorized as obese currently [22]. While obesity and CVD are certainly an epidemic for the adult population, there has also been an increase in the prevalence of both in the pediatric population, particularly in the state of West Virginia. Approximately $14.8 \%$ of the pediatric population in the US are categorized at obese, moreover, an estimated $20.9 \%$ of the pediatric population in $\mathrm{WV}$ is categorized as obese [52]. Further, recent work has shown that the students of West Virginia University (WVU) are at a 4 fold greater risk for metabolic risk factors, when compared to other similar state based universities [53].

The Metabolic Syndrome (MetS) exists as a cluster of co-morbidities, which lead to an increased risk for cardiovascular related mortality. The guidelines that detail these co-morbidities are described in the National Cholesterol Education Program Adult Treatment Panel III (NCEP), which requires that at least three of five guidelines be met. These guidelines are abdominal obesity, hypertension, hyperglycemia, hypertriglyceridemia, and decreased high density lipoprotein (HDL) [25]. MetS leads to an increased risk for CVD, when compared to healthy individuals. The presence of MetS is associated with a 3-fold increase in the risk for a cardiovascular incident, including myocardial infarction and cerebrovascular accident [1]. There is conflicting evidence 
regarding the underlying mechanism for this increased risk [26]. Studies have shown that the development of MetS is associated with an increase in the stiffness of the large conduit arteries, such as the aorta [26-28]. Other studies have shown that the development of MetS is associated with endothelial dysfunction [26, 28]. It is likely that there is interplay between both stiffness of the arteries and endothelial dysfunction, which culminate in the arterial detriments associated with MetS.

The young adult body, even unhealthy, is better able to adjust and rebound from the detriments of a poor diet and sedentary lifestyle. It has been shown that beta adrenergic receptors, which are related to blood pressure are less effective as humans age [54]. Although, there has been minimal research investigating dietary intervention in a young adult age group and the effects on AS. Therefore, it is of importance to determine proof of principle in utilizing dietary intervention for PWV in a young adult population.

In children with both hypertension and obesity, reported prevalence rates of MetS have been between $28-35 \%[52,55]$. This statistic is alarming, given the major health implication associated with MetS and illustrates the importance of elucidating a method to encourage the pediatric population to engage in activities and diets that have been shown to improve the risk factors associated with MetS. There are two major nonpharmacologic methods through which these risk factors can be ameliorated, which are dietary changes and exercise. Exercise has been shown to be an effective method to improve these risk factors, however, less than $50 \%$ of adults in WV meet the 150 minutes of exercise that is recommended per week and approximately $30 \%$ of adults perform no exercise [56]. Also, only $31 \%$ of adolescents exercise daily thus the 
population of $\mathrm{WV}$ is not meeting exercise goals, therefore another method of action should be elucidated [56].

Poor diet has been associated with arterial stiffness, particularly diets high in fats, glucose, and sodium [57-59]. These dietary elements function through different mechanisms to culminate in arterial stiffness. Similar to exercise, young adults are not consuming appropriate diets. In 2010 , only $28 \%$ of young adults consumed the appropriate amount of vegetables, while $94 \%$ also exceeding the recommendations for empty calories [60]. Diets with elevated fat content have been shown to increase pulse wave velocity and arterial wave reflection, measures of arterial stiffness, 4-hours postprandial of a meal high in either mono-unsaturated fatty acids or saturated fatty acids, in healthy men [59]. Similarly, diets high in glucose have been implicated in contributing to the development of AS. Diets high in simple carbohydrates have been shown to increased arterial stiffness through elevations in blood pressure, increases in inflammatory cytokines, and through decreased nitric oxide bioavailability [31, 61]. Thus, a diet high in fruits and vegetables would likely exert a beneficial action on vascular function, given that a diet composed of fruits and vegetables has been shown to improve microvascular reactivity [2]. The primary aim of this study was to assess a diet consisting of $50 \%$ of fruits and vegetables over 8 weeks on measures of arterially stiffness, while secondarily the aim was to assess if a low carbohydrate or low fat component in addition to this F\&V diet would exert a greater benefit.

The aim of the present study was to examine the complex relationship between diet and vascular function, in a population of college aged students in WV. We 
hypothesize that increasing fruits and vegetables, while reducing the consumption of simple carbohydrates and saturated fatty acids, will lead to improved vascular function. 
Methods:

For the FRUVEDomics study we recruited 37 college aged individuals (age $=23 \pm 0.4$, who were randomly assigned into 3 groups, Fruved $(n=12)$, Fruved + low refined carbohydrate $(n=13)$, and Fruved+ low fat $(n=12)$. We had 1 participant drop out of the study after the baseline measures. The base diet for all of the groups was the Fruved diet, in which $50 \%$ of the food consumed must be fruit or vegetable, which follows the United States Department of Agriculture (USDA) Dietary Guidelines for Americans (DGA) 2015. The other two groups followed the same base diets, but had additional stipulations of either low refined carbohydrate or low fat. The low refined carbohydrate was focused on decreasing refined carbohydrates and advising the participants to choose low-glycemic foods and limiting intake of high-glycemic foods [62]. Low glycemic load diets have previously been shown to reduce total cholesterol, while also increasing HDL cholesterol [63]. Contrastingly, high-glycemic load diets have been shown to increase inflammatory processes in the body and increase total cholesterol [63]. The low fat diet was based on the American Heart Association (AHA) guidelines [64], which show that $30 \%$ of the calories consumed are from fats. These guidelines restrict that saturated fats comprise only $10 \%$ of this diet, only $30 \mathrm{mg}$ of cholesterol is consumed daily, and advise little to no consumption of trans fats. All three of the interventions took place over the same period of 8-weeks. Overall, 36 participants completed the intervention timeline. Participants were asked to maintain their current level of physical activity and lifestyle aside from the study diet. The exclusion criteria for the study are as follows: 1) pregnant, 2) Antibiotic use in the last 6 months, 3) hospitalized within the last 6 months, 4) Suggestion of eating disorder (ED) or any 
treatment for an ED in the past 1 year, 5) $\mathrm{BMl}<18$ or weight $<110 \mathrm{lbs}, 6$ ) Alcohol or drug abuse within the past year, 7) Taking psychotic medications or history of major psychiatric disorder, 8) Major medical diseases, 9) Taking any medication for GERD, Metformin, or Betablockers. The inclusion criteria were broad and as follows: 1) 18-28 years old, 2) Currently consuming a poor diet. All subjects provided written informed consent to participate, which was submitted to and approved by the West Virginia University Institutional Review Board.

\section{Study Design}

Assessments were all performed in a temperature controlled room, following a 12 hours fast from food, as well as abstinence from alcohol, caffeine, and vitamins. Participants were brought into a quiet room, where they were placed in supine position on an assessment bed and the lights were dimmed. The participants maintained in supine position and rested for 15 minutes prior to any assessments. Following the rest period, the measurements of arterial structure, arterial function, body composition, and a blood draw were all performed.

\section{Body Anthropometry}

Participants underwent height, weight, and hips to waist circumference ratio, which were all performed using standard laboratory procedures. The hip/waist circumference measurements were performed to assess fat distribution; the waist circumference was measured at the smallest area between the rib cage and iliac crest and the hip circumference was measured at the largest area between the waist and the 
thighs. Body composition was measure with air displacement plethysmography, by the BodPod $®$ system (Life Measurement, Concord, CA, USA). Body Mass Index (BMI) was calculated with the formula weight $(\mathrm{kg}) /$ height $(\mathrm{m})^{2}$. In addition, physical activity (PA) was assessed dually. First with an accelerometer and then with an International Physical Activity Questionnaire (IPAQ), which were both performed at baseline and again the final week of the study intervention.

Blood Analysis

Venous blood samples were obtained following a 12-hour fast. Samples of serum were obtained from blood samples that were collected in serum separation tubes with polymer gel/silica activator (BD Vacutainers), while the plasma samples were obtained from blood samples that were collected in plasma separation tubes (BD Vacutainers). Standard laboratory procedures were followed to obtain total cholesterol, HDL cholesterol, LDL cholesterol, triglycerides, insulin, and glucose levels. These were all processed at the West Virginia University Hospital's central laboratory in Morgantown, WV.

\section{Arterial Geometry}

Ultrasound (GE Vivid i) images of the right common carotid artery (CCA) were obtained $1-2 \mathrm{~cm}$ proximal to the carotid bifurcation. Both the posterior and anterior intima media were visualized, in order to obtain measures of maximal lumen diameter and carotid intima media thickness (cIMT). 
Arterial Function

Systolic and diastolic blood pressures (SBP and DBP) were obtained at the brachial artery, via an automated sphygmomanometer (Critikon Dinamap Compact BP monitor, GE Medical Tampa FI, USA). Pulse pressure (PP) was obtained by the formula: SBP-DBP. Pulse wave analysis was measured, in triplicate, at the right radial artery, via applanation tonometry (SphygmoCor System, AtCor Medical, Sydney, Australia). This device is able to generate central (aortic) SBP and DBP, using the wave reflection detected. This is accomplished through the use of a validated general transfer function, and these generated measurements have been shown to be identical to intraarterially measured pressures $[65,66]$. The Buckberg subendocardial viability ratio (SEVR) is also derived from radial applanation tonometry. SEVR is a ratio of oxygen supple to oxygen demand of the myocardium. SEVR is calculated as a ratio of diastolic pressure time index to systolic pressure time index [67].

Carotid to femoral pulse wave velocity (cfPWV) was measure via simultaneous capturing of the carotid arterial pulse wave form, obtained via applanation tonometry and the femoral pulse waveform, obtained via volume displacement in an inflated cuff placed on the right upper thigh (Xcel System, AtCor Medical, Sydney, Australia). Measurements were obtained from the suprasternal notch to the carotid artery measurement site, from the suprasternal notch to the cuff of the upper thigh, and from the femoral artery to the cuff on the upper thigh. The device utilizes these measurements and the difference in time from the onset of the diastolic foot in both the carotid and femoral measurements. This method was validated to yield similar results 
to traditional PWV measurements utilizing sequential carotid and femoral measurements via applanation tonometry with gated EKG [68].

Intervention

All of the participants that enrolled to participate initially met in a classroom setting with a registered dietitian nutritionist (RDN), where they were educated on proper food choices, portion control, smart and healthy shopping, cooking techniques, among many other topics. The participants were then provided an culinary toolkit, which included essentials to cooking healthfully, these included cutting board, knives, liquid measuring cups, dry measuring cups, peeler, can opener, containers, and baggies. Before the commencement of the intervention all of the subjects will attend a group education session, which is used to introduce the specific diet, to which they were assigned. The RDN will conduct the education session and instruct the participants on the basic aspects of nutrition and prescribing to a diet.

12 participants were randomly placed into each of the three groups, Fruved, Fruved +low refined carbohydrate, and Fruved +low saturated fat. The participants of each group were advised on food that would benefit their diet and food that should be avoided for their specific diet. At baseline, the participants all completed an Automated Self-Administered 24 Diet Recall (ASA-24), which was used to determine the baseline diet of all of the participants. Weekly, during the study, the participants were asked to log their food in a journal, take pictures of their meals, and keep food receipts (grocery and restaurant), which were all analyzed to track the food consumption. These methods were used in conjunction to control for food log bias, in an attempt to ensure that the 
participants logged all of their meals and limit any self-report bias. All participants were also required to meet with an RD weekly throughout the study duration to discuss their progress and advice was given on how to further improve their diet. The RDN computed the self-report food log in a nutrition analysis software (Nutritionist Pro, Version 4.5.0, 2010, Axxya Systems, Staffard, TX, USA), which was used to determine various nutritional components in the diet of the participants. The RDN also recorded a compliance to diet score weekly, as well as questioning the participant regarding any major events that may have impacted their diet for that week of time. Additionally, the participant will rate how they feel that their compliance has been over the past week. Finally, the participants will rate their hunger during the diet on a scale of 1-5 and they will note any changes in their bowel movements.

The use of digital photography has previously been used to assess nutritional compliance, and helps the participants accurately recall what and how much of a meal was prepared and consumed $[69,70]$. This will help the RDN accurately input the information into the nutrition analysis software, while being minimally disruptive to the daily life of the participants, particularly when compared to direct supervision and observation. The incorporation of these methodologies and the utilization of mobile devices help to make these free living conditions feasible.

Free living dietary interventions may be considered flawed and less controlled than a direct observation intervention, however, it is more representative of true daily life and makes the findings of this study more translational. 


\section{Statistical Analysis}

Repeated measure ANOVA was used to analyze significant effects of dietary intervention on arterial parameters, with Tukey's post hoc test. All analyses were performed with the use of SPSS version 22 (SPSS, Chicago, IL). Values depicted in the tables are representative of means \pm standard error, unless otherwise noted.

Significance was defined as $P \leq 0.05$. 
Results:

Overall, 36 participants were recruited and placed into the 3 groups. There were no significant interaction in age, weight, BMI, bSBP, bDBP, and baseline arterial parameters between groups, these characteristics of the cohort are illustrated in Table 3.1. We began the study with 37 participants, after the baseline measurements we had 1 participant dropout of the study, thus our attrition rate was $2.7 \%$. When evaluating the participant's compliance to the diet, it was determined that 30 participants were considered compliant. There were no significant changes in any of the groups from pre to post in terms of physical activity ( $p=n s)$.

\section{Dietary Changes}

Participants met with a RDN weekly, where their food logs, food pictures, food receipts and diet were analyzed and discussed using motivational interviewing technique. We found that the first week of the intervention had a major impact on the total calories (TKcal) consumed, with a $16 \%$ reduction in all groups combined. While individually, Fruved decreased $26 \%$, Fruved + Low $\mathrm{CHO}$ decreased $21 \%$, although Fruved+ Low Fat increased 1\%. Following this initial decrease there was a plateau, where participants maintained their TKcals at approximately the same level from week 3 on. We also investigated the changes in empty calories, which showed with all groups combined there was a $46 \%$ decrease between the baseline measurements and the end of the first week of the intervention. When separated into the 3 groups, Fruved had a $53 \%$ decrease, Fruved +Low refined $\mathrm{CHO}$ had a $51 \%$ decrease, and Fruved + Low Fat had a $27 \%$ decrease in their empty calories, which are calories from solid fat and added 
sugar [71] (Figure 3.1). As the intervention proceeded, a steady decline in the empty calories continued. Finally, we investigated how consumption of fruits and vegetables changed throughout the intervention. With all groups combined there was an increase of $89 \%$ in consumption of fruits and vegetables. Separately the groups, Fruved showed an increase of $119 \%$, Fruved +Low refined $\mathrm{CHO}$ increased $50 \%$, and Fruved + Low Fat increased 104\% (Figure 3.2).

The RDN analyzed the participant's food consumption weekly, throughout the study period and gave compliance scores. Further, it was deemed that 30 of the 36 participants who completed the study were compliant with the diet.

\section{Arterial Stiffness}

Eight weeks of dietary intervention improved the Buckberg Sub-endocardial variability ratio (SEVR) with a significant time interaction $(P \leq 0.001)$ (Figure 3.3). Importantly, bSBP and cSBP were also significantly reduced following intervention $(P \leq 0.001, P=0.013$ respectively, Figure 3.4$)$. Contrastingly, 8 weeks of dietary intervention produced no significant changes in cfPWV in or between any of the groups (Figure 3.5). Further, when eliminating those that were deemed non-compliant to the diet, cSBP, bSBP, and SEVR maintained significance $(p=0.009, p=0.006$, and $p=0.003$ respectively). Unfortunately, even eliminating those that were non-compliant with the diet, none of the measures of AS reached significance. 
Blood Analysis

We also examined the changes in various $\mathrm{CV}$ risk factors present in the blood in response to the dietary intervention. There were no significant differences in triglycerides, total cholesterol, LDL, glucose, and insulin. However, there was a significant time interaction for HDL levels $(P=0.003)$. Table 3.3 illustrates the values for the changes in the blood values. 


\section{Discussion:}

It has been postulated that elevation in arterial stiffness often occur prior to the onset of metabolic manifestations of the CVD, particularly hypertension [72]. Therefore, we sought to evaluate the effects of a dietary intervention on a younger adult population at risk for the development of MetS. Given the great number of side effects that can be associated with pharmacological treatments of cardiovascular risk factors, the first line of treatment should be a health promoting dietary modification [73]. The best option is to utilize preventative treatment for cardiovascular risk factors, or to intervene at a young adult age, before the risk factors progress further and while the body is more resilient [74].

The main outcome of this study was based on changes in diet, with increases in fruits and vegetables, as well as two group components of limited refined carbohydrates or saturated fats. The first week of the intervention lead to significantly increased fruits and vegetables in all of the groups, with a combined $88.5 \%$ increase in fruit and vegetable consumption, rising from 2.26 cups per day to 4.26 cups per day, which steadily increasing throughout the intervention, with the highest amount of fruits and vegetables consumed being 4.92 cups in the final week of the intervention. While this increase is substantial, it is of note that this increase failed to raise the participants in the intervention to the USDA DGA recommendations of 5 cups of fruits and vegetables [75]. Although, the DGA recommendation is based on a 2000 calorie diet, which was the level of calorie consumption of our participants at the commencement of the intervention, but approaching the end of the intervention there was an average 
consumption of 1836 calories. With this considered, the recommendation would be based on an 1800 calorie diet, which is 4.5 cups of fruit and vegetable daily. In order to ensure that our results were a result of the dietary intervention, as opposed to other methods to improve arterial function, such as PA, we tested to ensure there were no changes in terms of PA. We found no significant changes in PA from pre to post.

In addition to the increasing of fruits and vegetables, we also found that there were decreases in total calories consumed and a decrease empty calories. Given that both empty and total calories had a decline, it could be associated with snacking shifting from empty calories foods to lower calorie fruits and vegetables.

Our results show that we significantly increased the consumption of fruits and vegetables in our participants over the course of 8 weeks. This is a population, which on average $78 \%$ report that they do not consume the recommended 5 servings of $F \& V s$ per day. This in and of itself is an accomplishment and given the known benefits of consuming F\&Vs, even outside of the CV system, shows promise even in lieu of changes in AS.

\section{Arterial Function}

Of importance, we report that 8 weeks of dietary intervention, regardless of reduction in refined carbohydrates or saturated fats, resulted in significant improvements in bSBP, which is the traditional SBP measurement at the brachial artery, and CSBP, which is a measure of the SBP at the aorta. We saw reductions in all groups, although the reduction was more pronounced in the Fruved + Low refined $\mathrm{CHO}$ group. We showed decreases in cSBP of over $2 \mathrm{mmHg}$ in the Fruved group, $6 \mathrm{mmHg}$ in the 
Fruved + Low CHO, and $3 \mathrm{mmHg}$ in the Fruved +Low fat groups. These results are clinically significant, given that reductions in SBP of $2 \mathrm{mmHg}$ have been correlated to a decreased risk of stroke-related mortality of $10 \%$ and a decreased risk of vascularrelated mortality by $7 \%[76]$. The question then becomes, what elicited such a substantial decrease in the Fruved +Low CHO group? Upon further investigation, the Fruved +Low refined $\mathrm{CHO}$ also had a change in $\mathrm{PWV}$ from $6.06 \mathrm{~m} / \mathrm{s}$ to $5.74 \mathrm{~m} / \mathrm{s}$ ( $p=n s$ ), given the complex relationship between BP and PWV these are likely related. Additionally, the Fruved + Low refined $\mathrm{CHO}$ showed a decrease in triglycerides, while the other two groups resulted in minor increases. We showed no significant changes in PWV, which is likely due to the fact that our participants were young adults with healthy AS values, therefore, there was minimal room for improvement.

Recent findings have shown that a low refined carbohydrate and high fat diet was associated with decreased SBP, which the authors postulate results from improved reactivity of small arteries of resistance [77]. Animal models have shown that high fat and refined carbohydrate diet are associated with HTN and decreased NO bioavailability [78]. This reduced NO bioavailability was associated with reactive oxygen species (ROS), which can inactivate NO. Interestingly, we did not elicit any changes in the bDBP or cDBP in our participants. This could potentially be explained by their low initial DBP values, again leaving minimal room for improvement.

\section{Future Directions}

Our results show that there were no significant changes in measures of arterial stiffness following 8 weeks of dietary intervention in this age group. Given this, it may be 
of interest to investigate the dietary intervention over the course of a longer period of time, given the that the changes in diet did not take place immediately at the commencement of the intervention, instead they had a sharp change then a steady climb to reach the appropriate level toward the end of the intervention. Therefore, perhaps a 12-week or even longer intervention could show improvements in arterial stiffness measurements.

Aerobic exercise has been shown to be an effective modulator of AS, with our lab previously showing that 8-weeks of aerobic activity significantly decreased PWV in adults with MetS [1]. Investigating the effects of combined aerobic exercise and nutritional intervention would be an interesting avenue of research. Perhaps in the setting of exercise a diet high in fruits and vegetable, similar to that which was utilized in this intervention, could elicit a bolstered response, compared to exercise alone.

Additionally, fruits and vegetables with high levels of nitrite and nitrates, such as supplementation with beetroot juice, have been associated with transient reductions in PWV [41]. Therefore, making the dietary intervention more specific to fruits and vegetables with higher levels of nitrites/nitrates could yield results of improved AS, due to increased bioavailability of NO, which a potent vasodilator and major modulator of arterial tone [79].

Finally, the population that we utilized in this study was a young adult population, which often have lower PWV, given that PWV has been directly correlated to age [1]. Therefore, it would be of interest to utilize this intervention in an old healthy population and also those afflicted with MetS. Those with MetS have been shown to have elevated PWV, therefore, would be more likely to glean benefits from this intervention [28]. 


\section{Limitations}

All studies are with limitations; the present study is of no exception. To begin there was no true non-intervention control group, to control for time changes, although, we did use a pre to post self-control method. This can have major implication on the interpretation of data, given that there is no group without intervention for comparison. Additionally, another major source of limitation is variability in the techniques of PWV and pulse wave analysis.

Another major limitation in the study was associated with the diet intervention. Self-report diet logging in general is a limitation, given that it is quite easy for the participants to withhold some of their consumption or give false reports in the amount of food consumed. In addition, the amount of fruits and vegetables consumed by the completion of the study was not increased to the level anticipated. Finally, the length of the diet could be a limitation. The 8-week intervention lead to reductions in bSBP and cSBP, blood pressure can be a major modulator in arterial stiffness. Given this, a prolonged intervention period may have led to improvement in measures of AS. 
Table 3.1: Clinical Characteristics of the Cohort

\begin{tabular}{|c|c|c|c|}
\hline & FRUVED & $\begin{array}{l}\text { FRUVED + LOW } \\
\text { REFINED CHO }\end{array}$ & $\begin{array}{c}\text { FRUVED +LOW } \\
\text { FAT }\end{array}$ \\
\hline AGE, YR & $24 \pm 0.45$ & $22 \pm 0.77$ & $24 \pm 0.61$ \\
\hline SEX, \%FEMALE & $58 \%$ & $54 \%$ & $58 \%$ \\
\hline HEIGHT, CM & $171 \pm 1.64$ & $169 \pm 3.42$ & $172 \pm 3.47$ \\
\hline WEIGHT, KG & $81 \pm 4.57$ & $78 \pm 6.40$ & $81 \pm 7.92$ \\
\hline $\mathrm{BMI}, \mathrm{KG} / \mathrm{M}^{2}$ & $28 \pm 1.51$ & $27 \pm 2.11$ & $27 \pm 1.52$ \\
\hline BODY FAT, \% & $30 \pm 3.75$ & $29 \pm 3.08$ & $27 \pm 3.00$ \\
\hline WAIST CIRCUMFERENCE, CM & $85 \pm 3.87$ & $84 \pm 4.19$ & $86 \pm 4.01$ \\
\hline
\end{tabular}

Values are means \pm SE. BMI, body mass index.

Table 3.2: Changes in Arterial Parameters Following Fruved Dietary intervention

\begin{tabular}{|l|c|c|c|c|c|c|}
\hline \multirow{2}{*}{} & \multicolumn{2}{|c|}{ Fruved } & \multicolumn{2}{c|}{$\begin{array}{c}\text { Fruved+ Low refined } \\
\text { CHO }\end{array}$} & \multicolumn{2}{c|}{ Fruved +Low Fat } \\
\cline { 2 - 7 } & Pre & Post & Pre & Post & Pre & Post \\
\hline HR (BPM) & $66.2 \pm 3.7$ & $59 \pm 3.2$ & $70 \pm 2.9$ & $66 \pm 2.9$ & $70 \pm 2.7$ & $67 \pm 3.5$ \\
\hline bSBP $(\mathrm{mmHg})$ & $121 \pm 5.4$ & $109 \pm 3.6^{*}$ & $112 \pm 3.6$ & $107 \pm 2.7^{*}$ & $116 \pm 3.8$ & $113 \pm 2.7^{*}$ \\
\hline bDBP $(\mathrm{mmHg})$ & $58 \pm 2.9$ & $62 \pm 2.8$ & $63 \pm 3.7$ & $59 \pm 2.1$ & $64 \pm 2.6$ & $61 \pm 2.0$ \\
\hline cDBP $(\mathrm{mmHg})$ & $60 \pm 2.6$ & $63 \pm 2.8$ & $65 \pm 3.6$ & $60 \pm 2.0$ & $65 \pm 2.6$ & $62 \pm 2.1$ \\
\hline bMAP $(\mathrm{mmHg})$ & $76 \pm 2.4$ & $77 \pm 2.6$ & $79 \pm 3.4$ & $74 \pm 2.1$ & $80 \pm 2.9$ & $77 \pm 2.1$ \\
\hline
\end{tabular}

Values are Means $\pm S E$. HR, heart rate; bSBP, brachial systolic blood pressure; bDBP, brachial diastolic blood pressure; cDBP, central diastolic blood pressure; bMAP, brachial mean arterial pressure. ${ }^{*}=$ Significant interaction from pre to post. 
Table 3.3: Changes in Blood Characteristics Following Fruved Dietary Intervention

\begin{tabular}{|c|cccccc|}
\hline \multicolumn{2}{|c}{} & \multicolumn{2}{c}{ Fruved } & \multicolumn{2}{c|}{ Fruved +Low Refined CHO } & \multicolumn{2}{c|}{ Fruved+ Low fat } \\
\hline \multirow{2}{*}{$T G(\mathrm{mg} / \mathrm{dL})$} & Pre & Post & Pre & Post & Pre & Post \\
\cline { 2 - 7 } Chol $(\mathrm{mg} / \mathrm{dL})$ & $91.3 \pm 9.2$ & $96.1 \pm 9.1$ & $103.0 \pm 10.4$ & $89.1 \pm 12.1$ & $83.3 \pm 9.0$ & $92.8 \pm 14.4$ \\
HDL $(\mathrm{mg} / \mathrm{dL})$ & $179.5 \pm 8.8$ & $165.8 \pm 8.1$ & $179.3 \pm 8.5$ & $180.3 \pm 7.8$ & $175.4 \pm 7.6$ & $173.3 \pm 8.2$ \\
LDL $(\mathrm{mg} / \mathrm{dL})$ & $58.2 \pm 4.0$ & $49.7 \pm 3.5^{*}$ & $55.7 \pm 4.4$ & $53.9 \pm 4.6^{*}$ & $54.5 \pm 3.6$ & $51.2 \pm 3.0^{*}$ \\
Glu $(\mathrm{mg} / \mathrm{dL})$ & $103.0 \pm 6.6$ & $96.8 \pm 5.6$ & $103.1 \pm 7.3$ & $108.5 \pm 7.3$ & $104.3 \pm 5.7$ & $103.5 \pm 6.9$ \\
Insulin $(\mathrm{mg} / \mathrm{dL})$ & $80.2 \pm 1.3$ & $88.6 \pm 2.2$ & $85.1 \pm 1.5$ & $84.3 \pm 2.0$ & $86.1 \pm 3.2$ & $89.3 \pm 3.4$ \\
& $8.6 \pm 1.2$ & $8.4 \pm 1.3$ & $9.0 \pm 2.3$ & $8.2 \pm 2.5$ & $6.5 \pm 1.1$ & $10.0 \pm 2.4$ \\
\hline
\end{tabular}

Values are means \pm SE. TG, Triglycerides; Chol, Cholesterol HDL, High density lipoprotein; LDL, low density lipoprotein; Glu, Glucose. *= Significant interaction from pre to post.

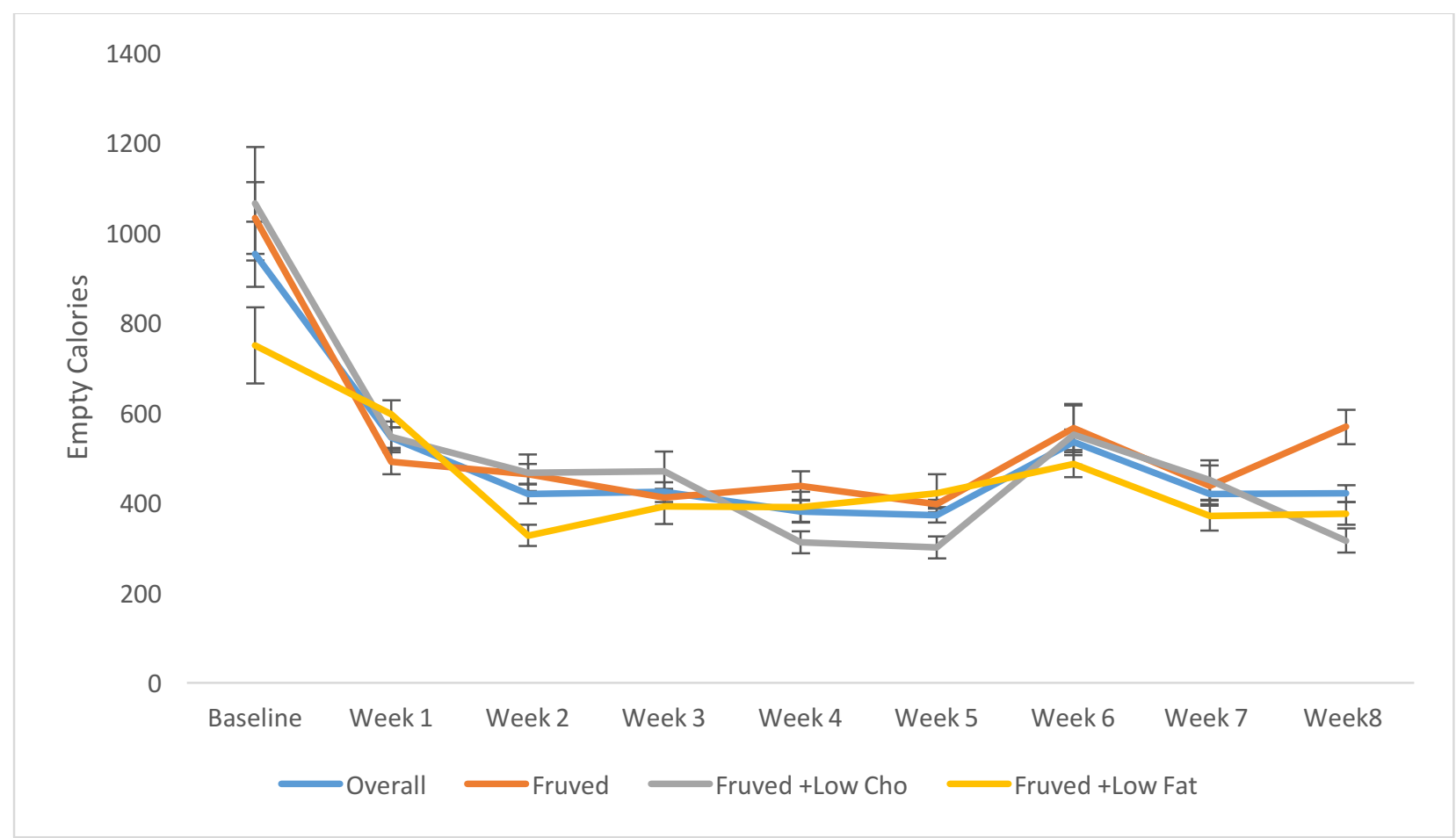

Figure 2.1: Changes in Empty Calories following 8 weeks of dietary intervention. There was a reduction in empty calories over the course of the intervention. 


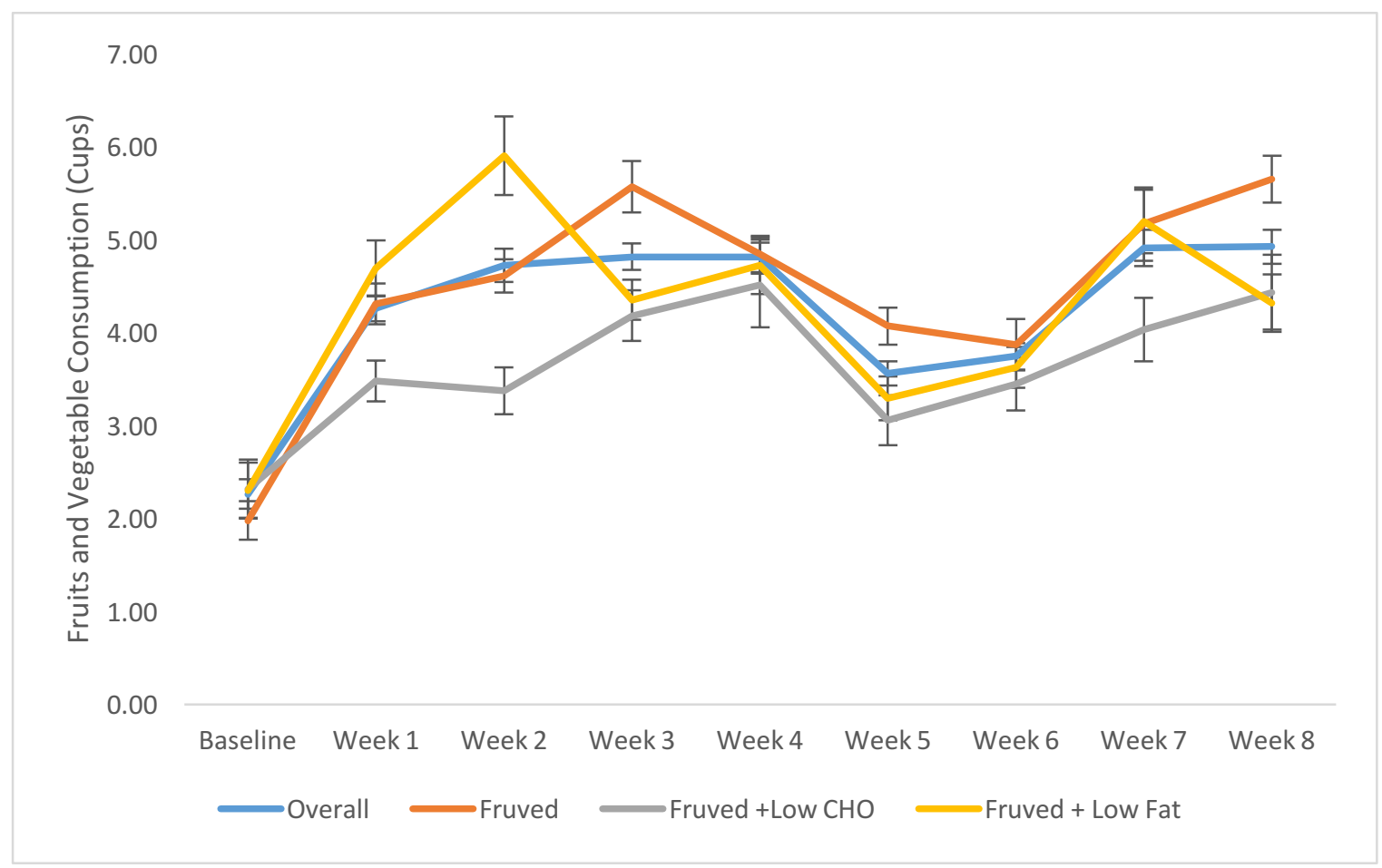

Figure 3.2: Changes in Fruit and Vegetable Consumption. There was a substantial increase in fruits and vegetables over the course of the intervention.

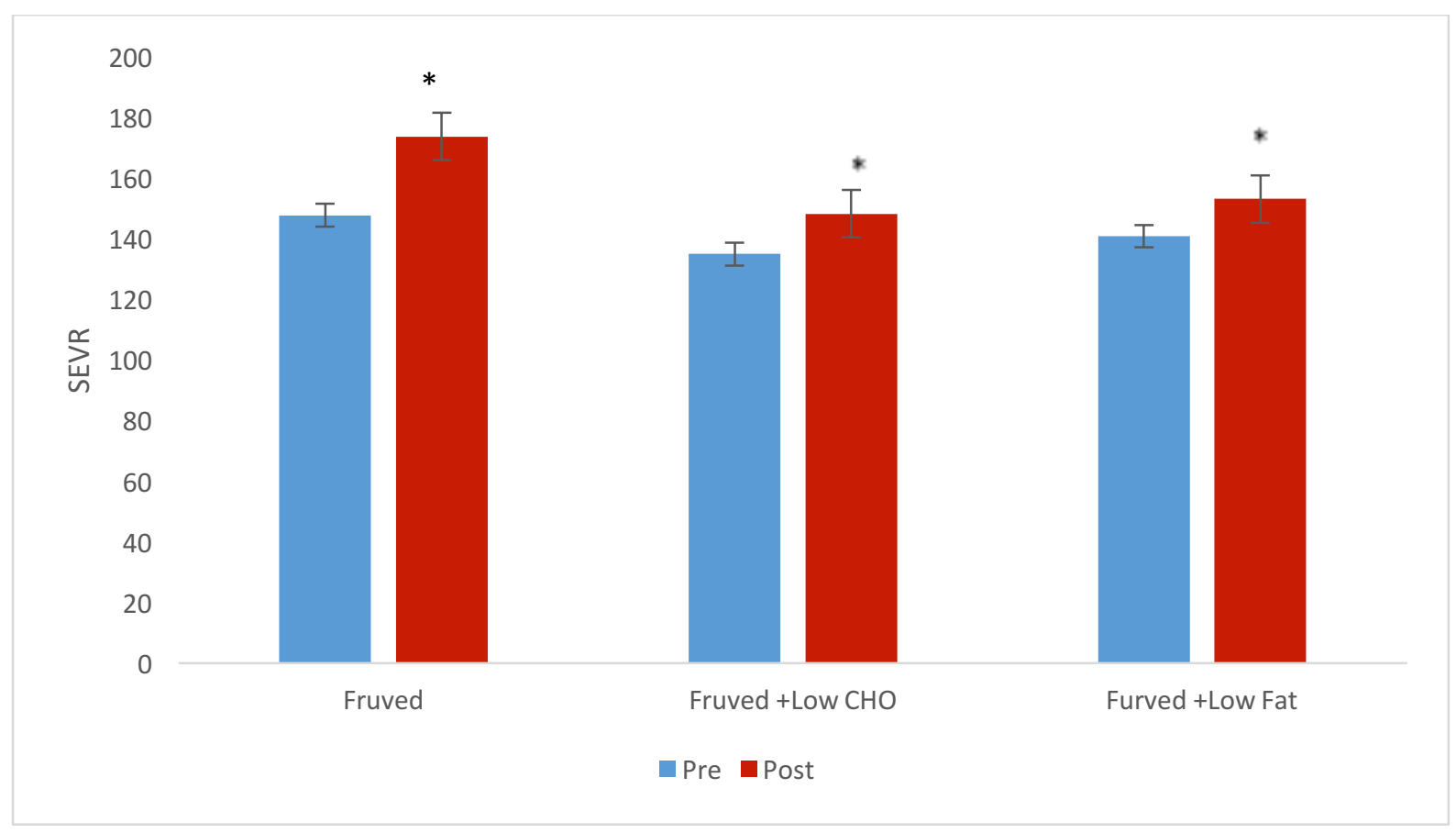

Figure 3.3: Changes in the Buckberg SEVR following Fruved dietary intervention. *= significant time interaction from pre to post. There was a significant improvement in SEVR in all groups over the course of the intervention. 


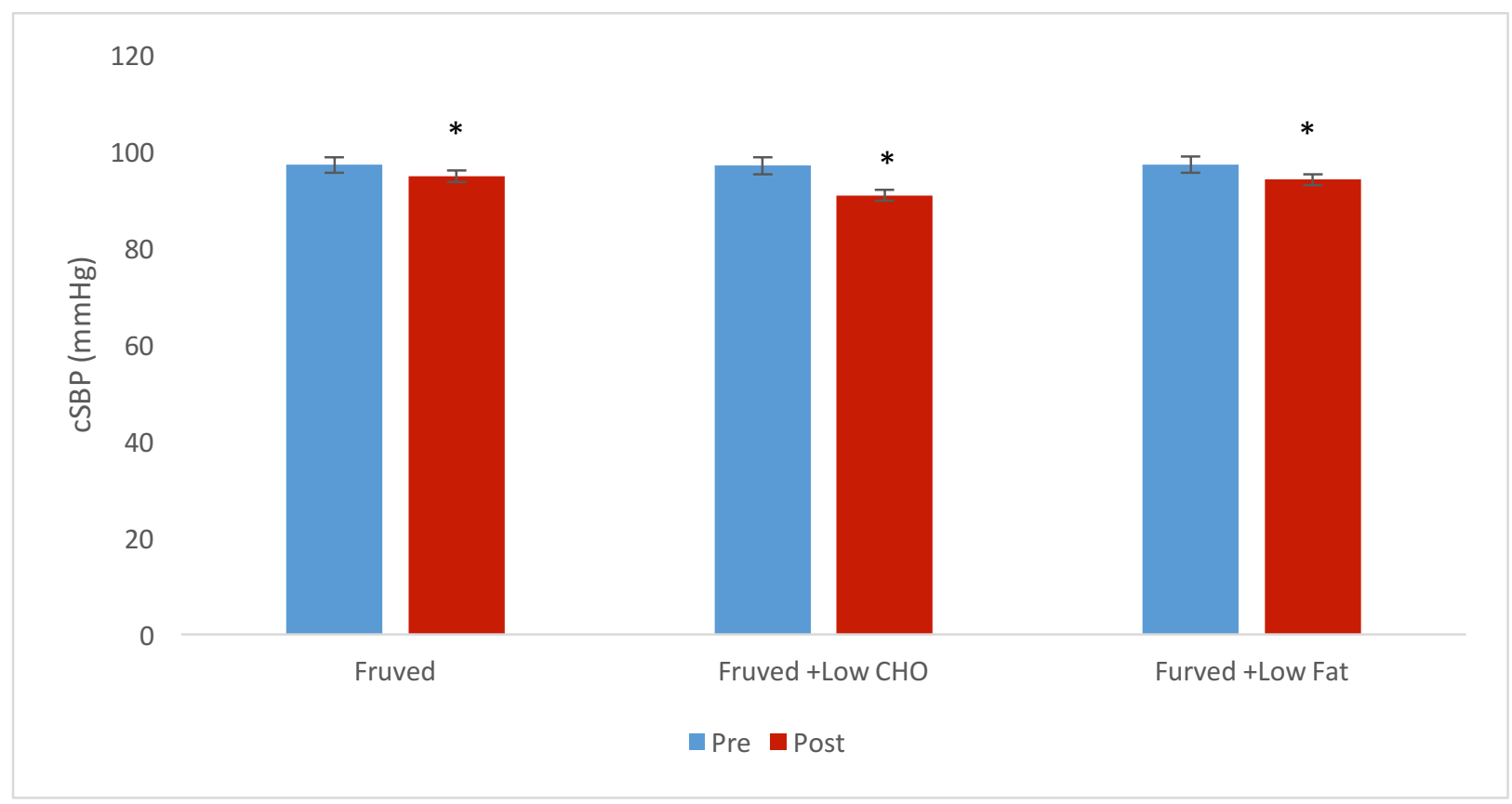

Figure 3.4: Changes in Central Systolic Blood Pressure (CSBP). There was a significant reduction in cSBP in all groups over the course of the interventions. ${ }^{*}=p>0.05$.

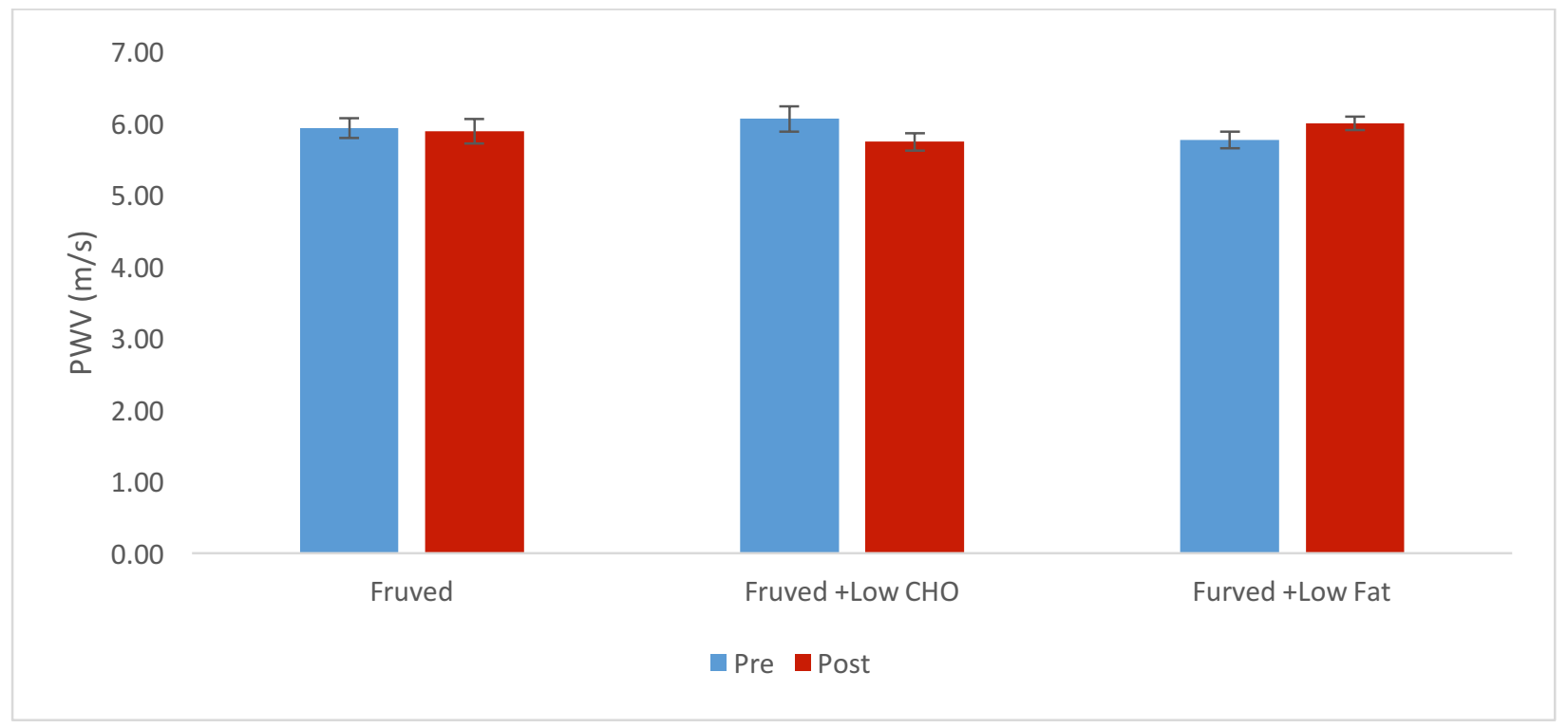

Figure 3.5: Changes in Carotid to Femoral Pulse Wave Velocity (PWV) following Fruved Dietary Intervention. There was no significant change in PWV over the course of the intervention. 


\section{Chapter 4: Is Deep Water}

\section{Aquatic Exercise an Effective}

\section{Exercise Modality to Improve}

Arterial Stiffness in Women

\section{with Metabolic Syndrome? A}

\section{Pilot Study}


Introduction:

Metabolic Syndrome (MetS) portends to a substantially increased risk of mortality associated with CVD, which has been estimated to be around a 3-fold increase [28]. Approximately $35 \%$ of all adults and up to $50 \%$ of adults over the age of 60 are estimated to have MetS [80]. There are a myriad of risk factors associated with the development of MetS, which, as defined by the United States National Cholesterol Education Program Adult Treatment Panel III (NCEP), necessitates the confirmation of three of five risk factors, which include: abdominal obesity, elevated triglycerides, decreased high density lipoprotein (HDL), hypertension (HTN), and hyperglycemia [81]. The increased CV risk that associated with MetS has previously been related to the increased arterial stiffness and central pressures, which naturally occurs with age, but is exacerbated by poor diet and a sedentary lifestyle [28, 57-59]. Arterial stiffness is measured non-invasively via pulse wave velocity (PWV), with an increase in PWV corresponding to an increase in CVD mortality risk of $15 \%$ [82].

Arterial stiffness is characterized by the increased deposition of collagen, decreased elastin, and increased carotid intima media thickness (cIMT). Mechanistically, stiffening can occur through two major pathways, structural stiffening or functional stiffening, or more simplistically vessel stiffening or vessel endothelial dysfunction [28]. Vessel stiffening typically occurs through increased strain on the vessels, which can lead to breakdown of the collagen fibers. These fibers take a particularly long time to degrade completely, as such these broken ends are often glycated together; these are called advanced glycation end products (AGE). Unfortunately, these AGEs are often formed in a markedly unorganized fashion, leading 
to further increases in the stiffness and dysfunction of the vessel [26]. Contrastingly, endothelial dysfunction occurs through errors in the signal pathway leading to vasodilation of the vessels. Notably, there is a reduction in the bioavailability of nitric oxide (NO), which is a potent vasodilator and the most noted modulated of vascular tone [79]. Many facets of endothelial dysfunction exist, which all culminate in the decreased bioavailability of NO. Remarkably, endothelial dysfunction is accentuated by a pro-inflammatory state, which is often associated with MetS. Circulating inflammatory molecules, such as C-reactive protein and interleukin-6 (IL-6) decrease the sensitivity of the endothelium to NO and they also may be implicated in increased contrary modulators of arterial tone such as endothelin-1 [28, 83].

Land based aerobic exercise, both chronic and acute, has previously been identified as an effective method for improving arterial health [1, 84-86]. While there are many benefits to land based aerobic exercise, in a population of middle aged to older adults with MetS there may be greater benefit to aquatic based exercise. Being placed in an aquatic environment, with no further activity, has been shown to decreased resting heart rate (HR) by approximately 12 beats per minute (BPM) [46]. This decreased HR is due to the hydrostatic pressure that is exerted on the entirety of the body by the water, which leads to increased venous return, increased stroke volume, and consequently decreased work of the heart to pump blood throughout the circulation [87]. Exercising in shallow water as well as swim base exercise have both been previously shown to improve PWV significantly and at a comparable level to land based exercise [6, 49]. PWV reductions are often accompanied with a reduction in central and peripheral blood pressure, therefore a reduction in central blood pressure may precede changes in AS. 
There has been minimal investigation of how aquatic exercise, particularly deep water aquatic exercise, effects vascular function in those with MetS. The aim of our study was to investigate how deep water exercise augments hemodynamics and CV function in a population with MetS. We hypothesize that deep water aquatic exercise, will lead to improvements in arterial stiffness, when compared to age and health matched controls. 
Methods:

We recruited 21 women (age $=57 \pm 3$ ) who met the criteria for MetS to participate in the study. These 21 women were placed into either the non-exercise control group $(n=11)$ and the aquatic exercise group $(n=10)$. The control group was asked to maintain their normal lifestyle with no changes in diet or exercise habits throughout the course of the 8 weeks of the intervention. Conversely, the aquatic exercise was asked to attend an aquatic exercise class 3 times a week for 8 weeks. Participants were asked to maintain the same nutrition level throughout the course of the intervention. Overall, we had 20 participants complete the intervention, therefore there was only $1 \mathrm{drop}$ out, which was from the aquatic exercise group. The exclusion criteria for this study were: 1) Subjects with inadequately controlled hypertension (i.e., screening blood pressure mandating initiation of antihypertensive therapy with BP greater than 160/100;2) A change of vasoactive medication or lipid-lowering medication in the past 2 months; 3) Inability to palpitate femoral or carotid pulses; 4) Diabetes mellitus treated with insulin or antidiabetic drugs or inadequately controlled diabetes on diet (hemoglobin A1C greater than $7 \%)$; 6) Inability to exercise or suspend vasoactive medicines for at least 24 hours; 7) Pacemaker or implantable cardiac defibrillators; 8) Subjects with contraindications to exercise will be excluded: i. Aortic stenosis ii. Symptoms of unstable angina iii. Myocardial infarction or coronary revascularization iv. Left Bundle Branch Block v. Resting heart rate greater than 120;9) Age less than 18; 10) Illicit drug or alcohol abuse; 11) Unexplained weight loss greater than $10 \%$ of body weight in past 6 months; 12) Anemia with hematocrit less than $32 \%$; 13) Pregnancy; 14) Cancer requiring treatment or recently completed treatment (last 2 months); 15) Reactive airway disease. 
All subjects provided written informed consent to participate, which was submitted to and approved by the West Virginia University Institutional Review Board.

\section{Study Design}

Assessments were all performed in a temperature controlled room, following a 12 hours fast from food, as well as abstinence from alcohol, caffeine, and vitamins. Participants were also asked to withhold their medications for 24 hours prior to the assessments. Participants were brought into a quiet room, where they were placed in supine position on an assessment bed and the lights were dimmed. The participants maintained in supine position and rested for 15 minutes prior to any assessments. Following the rest period, the measurements of arterial structure, arterial function, body composition, and a blood draw were all performed.

\section{Body Anthropometry}

Participants underwent height, weight, and hips to waist circumference ratio, which were all performed using standard laboratory procedures. The hip/waist circumference measurements were performed to assess fat distribution; the waist circumference was measured at the smallest area between the rib cage and iliac crest and the hip circumference was measured at the largest area between the waist and the thighs. Body composition was measure with air displacement plethysmography, by the BodPod $\circledast$ system (Life Measurement, Concord, CA, USA). Body Mass Index (BMI) was calculated with the formula weight $(\mathrm{kg}) /$ height $(\mathrm{m})^{2}$. 
Blood Analysis

Venous blood samples were obtained following a 12-hour fast. Samples of serum were obtained from blood samples that were collected in serum separation tubes with polymer gel/silica activator (BD Vacutainers), while the plasma samples were obtained from blood samples that were collected in plasma separation tubes (BD Vacutainers). Standard laboratory procedures were followed to obtain total cholesterol, HDL cholesterol, LDL cholesterol, triglycerides, insulin, and glucose levels. These were all processed at the West Virginia University Hospital's central laboratory in Morgantown, WV.

Arterial Geometry

Ultrasound (GE Vivid i) images of the right common carotid artery (CCA) were obtained $1-2 \mathrm{~cm}$ proximal to the carotid bifurcation. Both the posterior and anterior intima media were visualized, in order to obtain measures of maximal lumen diameter and carotid intima media thickness (cIMT).

\section{Arterial Function}

Systolic and diastolic blood pressures (SBP and DBP) were obtained at the brachial artery, via an automated sphygmomanometer (Critikon Dinamap Compact BP monitor, GE Medical Tampa FI, USA). Pulse pressure (PP) was obtained by the formula: SBP-DBP. Pulse wave analysis was measured, in triplicate, at the right radial artery, via applanation tonometry (SphygmoCor System, AtCor Medical, Sydney, Australia). As the heart contracts, a pulse wave is generated, which travels throughout 
the vasculature, but when this wave meets a bifurcation in the arteries a wave is subsequently reflected back toward the heart. In a young healthy individual, the pulse wave will travel more slowly due to the compliance of the arteries, therefore the reflection wave will occur later and will arrive back at the heart later in the contraction cycle, therefore it will arrive during diastole and increase the filling pressure of the ventricles. However, in an older individual or someone with CVD, the reflected wave will occur more quickly and may arrive at the heart during systole, which increased the pressure that the ventricle must contact against to eject blood. This device is able to generate central (aortic) SBP (cSBP) and DBP (dSBP), using the wave reflection detected. This is accomplished through the use of a validated general transfer function, and these generated measurements have been shown to be identical to intra-arterially measured pressures $[65,66]$. The device generates many values, including cSBP, cDBP, augmentation pressure (AP), augmentation index (AGI), and augmentation index adjusted to standard HR (AGIHR). AP is a measure of how the reflected wave impacts SBP, whether it increases the pressure the heart must contract against or if it increases the filling pressure of the ventricles. AGI, similar to AP, is a measure of how the reflected wave impacts SBP, but it differs from AP, given that it is relative to pulse pressure. AGI is often standardized to a specific heart rate, in most cases 75 beats per minute, and this variable (AGIHR).

Carotid to femoral pulse wave velocity, the gold standard measurement for AS, was performed while the participants were in the supine position, following 15 minutes of rest in a dark and quiet room. The measurements were obtained via applanation tonometry of the carotid and femoral arterials, in combination with voltage gated EKG 
(ATCor Medical, Sydney, Australia). Distances were measured from the suprasternal notch to probe placement at the femoral artery or from the carotid artery to the suprasternal notch. The difference of these were calculated and used by the computer program to calculate PWV. This calculation was generated by foot of the wave method. Intervention

Individuals that expressed interest in participating in this study were initially called and a phone screen was performed to evaluated whether they met the criteria to participate. If the participants met the criteria of the phone screen, they were invited to have a blood draw to determine if they met the criteria for MetS, which was outlined by the NCEP and 3 of 5 risk factors were required to be met. If all criteria were met, the participants were invited to participate.

They were placed in either the aquatic exercise group or the non-exercise control group. The non-exercise control group were asked to maintain their current lifestyle, with no modifications to diet, exercise, or medications. The aquatic exercise group were asked to attend 3 deep water exercise classes per week over the course of eight weeks. The deep water exercise classes were performed in a pool and the participants were given a floatation device that goes around their waste. The exercise was deep water jogging, which occurred for 60 minutes for each of the classes. The subjects were given polar HR monitoring devices. The participants exercise intensity was determined by their heart rate reserve (HRR). At the beginning of the training they were advised to keep their HR at $45 \%$ of their HRR, which increased over the course of the intervention to $75 \% \mathrm{HRR}$. It is of note that being in the aquatic environment can impact the HR due to increased venous return, this was accounted for by using the Kruel formula ( 220- 
age-(HR standing on land $-\mathrm{HR}$ standing in the water) $\times$ Desired exercise intensity percentage) to adjust land based HR to aquatic based.

Statistical Analysis

We utilized repeated measure ANOVA to analyze significant effects of aquatic exercise on arterial parameters, with Tukey's post hoc test. All analyses were performed with the use of SPSS version 22 (SPSS, Chicago, IL). Values depicted in the tables are representative of means \pm standard error, unless otherwise noted. Significance was defined as $P \leq 0.05$. Our total sample size is 20 , with only 9 in the intervention group. When a power analysis was performed, it showed that we needed 24 total, so 12 in each group, therefore our study is underpowered. 
Results:

In total, we have had 20 participants complete the study protocol, with 9 completing the aquatic exercise and 11 non-exercise controls. Therefore, the attrition rate was $4.8 \%$. These subjects were matched for age, weight, BMI, brachial systolic blood pressure (bSBP), brachial diastolic blood pressure (bDBP), central systolic blood pressure (cSBP), and central diastolic blood pressure (cDBP), with no significant differences between the groups (Table 4.1).

\section{Body Anthropometry}

Eight weeks of aquatic exercise training led to significant improvements in some measures of body anthropometrics. There was a significant time (pre vs post) by group (aquatics vs control) interaction for $\mathrm{BMI}$, with $\mathrm{P}=0.03$ (Figure 4.1). Additionally, this data illustrated a trend toward a decrease of body fat percentage (BF\%), with $\mathrm{P}=0.129$. Aquatic training showed a trend toward improvement in weight, with a time (pre vs post) by group (aquatic vs control) interaction $P=0.149$. This data was not able to illustrate any significant changes in body surface area (BSA), waist circumference (WC), or hip circumference $(\mathrm{HC})$, with $\mathrm{P}=0.592, \mathrm{P}=0.360$, and $\mathrm{P}=0.804$ respectively (Table 4.1).

Arterial Improvements

Eight weeks of aquatic exercise training in the population of this study was not able to show significance in any of the arterial measurements taken. While significance was not achieved, many of the measurements showed a trend toward improvement. $A G I$ and $A G I H R$, which are measures of arterial stiffness both showed a trend to 
improvement with $\mathrm{P}=0.180$ and $\mathrm{P}=0.212$ respectively (Figures 4.2 and 4.3 respectively). PWV, the gold standard method for assessing arterial stiffness, also showed a trend toward improvement, with $\mathrm{P}=0.144$ (Figure 4.4). The data also showed a trend for improvement in cSBP, with $\mathrm{P}=0.202$. The changes in arterial stiffness measure are illustrated in Table 4.2.

Blood Analysis

We measured changes in glucose, $\mathrm{HbA} 1 \mathrm{c}$, insulin, triglycerides, high density lipoprotein (HDL), and total cholesterol. There was a significant improvement in glucose, which showed a time (pre vs post) by group (aquatics vs control) interaction, with $\mathrm{P}=0.024$. We showed no significant changes in $\mathrm{HbA1c}$, insulin, triglycerides, $\mathrm{HDL}$, or total cholesterol (Table 4.3).

\section{Exercise Capacity}

Following 8 weeks of aquatic exercise training, there were trends toward changes in VO2. There was a trend towards a time by group interaction $\mathrm{VO} 2 \mathrm{max} \mathrm{P}=$ 0.120 , which trended toward improvement. There were no trends or changes in RER, VE, VT, or HRMax during exercise (Table 4.4). 


\section{Discussion:}

Metabolic syndrome is associated with increased risk for mortality, which is likely related to the elevated BP, increased arterial stiffness, and increased arterial tone [28]. These risk factors have previously been shown to be improved following aerobic exercise training [1]. However, traditional land based exercise is not tolerated well by all, those with MetS may have an increased joint pain during land based exercise due to gravity and their overall body habitus. Therefore, exercise drop-out rates are increased in this population of people. Aquatic exercise is a method of training that will lead to a decreased load on the joints, which can decrease the pain associated with joint load and perhaps increased exercise compliance [6]. We tested whether aquatic exercise training can improve MetS risk factors, exercise capacity, and arterial stiffness following an 8 week course of deep water exercise training.

\section{MetS Risk Factors}

There are many modifiable risk factors for the development of MetS, which can be augmented with exercise intervention. These include weight, BMI, circulating glucose, among other factors. We showed that aquatic exercise was able to significantly reduce $\mathrm{BMI}$ and also showed a trend toward improvement in weight and $\mathrm{BF} \%$. We did show a significant reduction in circulating blood glucose. Decreased circulating glucose can lead to decreased AS due to a reduced circulating insulin level, which can lead to decreased release of calcium, which a known vasoconstrictor [88]. Reduced insulin levels have also been shown to increase circulating magnesium, which is a modulator of vascular tone, leading to greater relaxation of the vessel [89]. There were no other 
changes in the other blood related risk factors of CVD, such as Triglycerides, $\mathrm{HbA} 1 \mathrm{c}$, HDL and total cholesterol, showed no statistically significance, which could possibly be related to the length of the study. Perhaps a longer intervention could elicit responses in these measures.

Arterial Function

Arterial stiffness is associated with substantial increased risk for CVD, and increasing AS by $1 \mathrm{~m} / \mathrm{s}$ has been shown to increase the CV and all-cause mortality by $15 \%$ [82]. We showed no significant changes in our primary outcome, PWV, between the aquatic exercise group and the non-exercise control group. We found similar nonsignificant findings in other measures of AS, AGI and AGIHR. Land based aerobic exercise has previously been shown to improve cfPWV, which is likely mediated through improved NO bioavailability [90]. Unfortunately, we were not able to show significance in this population with MetS, however, our subject total was very low, and we did not have enough subjects to ensure statistical power. Perhaps, with a greater number of subjects, we would be able to show significance.

PWV is also mediated through changes in peripheral and central blood pressure (CBP), which have also been shown to improve following land-based based exercise training [1]. However, we failed to elicit changes in cSBP, cDBP, bSBP, or bDBP following aquatic exercise training.

It is perplexing that the exercise intervention prescribed in this protocol did not elicit any changes in AS in a population with MetS. Upon further investigation, we found that there was not a significant improvement in $\mathrm{VO} 2$, which is a measure of 
cardiovascular fitness, therefore, perhaps the exercise intensity was not high enough, or the subjects were working at the proper intensity.

\section{Future Directions:}

Our results show no significant changes in measures of $A S, P W V, A G I$, and AGIHR. Our lab has previously shown significant improvement in PWV following 10 weeks of shallow water aquatic exercise training [6]. The present intervention took place over the course of 8 weeks, therefore a longer intervention may have been able to elicit this changes in AS.

Various dietary component have been shown effective at improving PWV in older or unhealthy populations $[15,91,92]$. Thus it is of interest to determine if dietary intervention is as effective as exercise intervention, or if there is a bolstered impact on PWV, when combining diet and exercise intervention. Perhaps, a diet high in nitrates would lead to decreased AS in the setting of exercise, given the increased NO bioavailability.

Another interesting addition for the future research could be to add additional measures, to further evaluate mechanisms of changes in AS. By reducing body fat it has previously been suggested that there are also reduction in Interleukin-6 (IL-6) and C-reactive protein (CRP) $[93,94]$. CRP and IL-6 have been shown to increase insulin resistance, which would lead to a CV risk. We did not directly measure IL-6 or CRP, These inflammatory molecules have been shown to be associated with increased AS. Perhaps, changes in these molecules in response to exercise could be another method 
to assess whether there are changes taking place at the cellular level, but simply have not had an effect on overall stiffness at the time of post measurements [95].

\section{Limitations:}

This study does have limitations that must be considered when evaluating the results and outcomes. One of the most stark limitations of this study is the sample size that we have attained. Our power analysis revealed that in order for the study to have effective power we would need a total sample size of 24 (or 12 in each the control and the intervention). We had only 11 in the control group and only 9 in the intervention group. The recruitment was very limited for our study, due to the time that the instructor was available to teach the classes, which was during the normal work day. This lead to difficulty recruit participants who were able to participate in the intervention. With a larger sample size, we postulate many of the trends toward improvement elicited, would reach statistical significance.

Additionally, it is of note that there was no log of dietary habits to ensure that there were no major dietary shifts throughout the course of the study, which could have major implications on AS. If the subjects inadvertently increased their consumption of omega-3 fatty acids, nitrates, or any other vasoactive components that are often present in diet, then these could have at least partially impacted the results that we found.

Finally, we had no measure of what was occurring on a cellular level, therefore we can only speculate as to the underlying mechanism for the improvement that we found. If we were to measure inflammatory markers, then perhaps we would be able to better explain the underlying mechanism of the improvements in PWV and BP. 


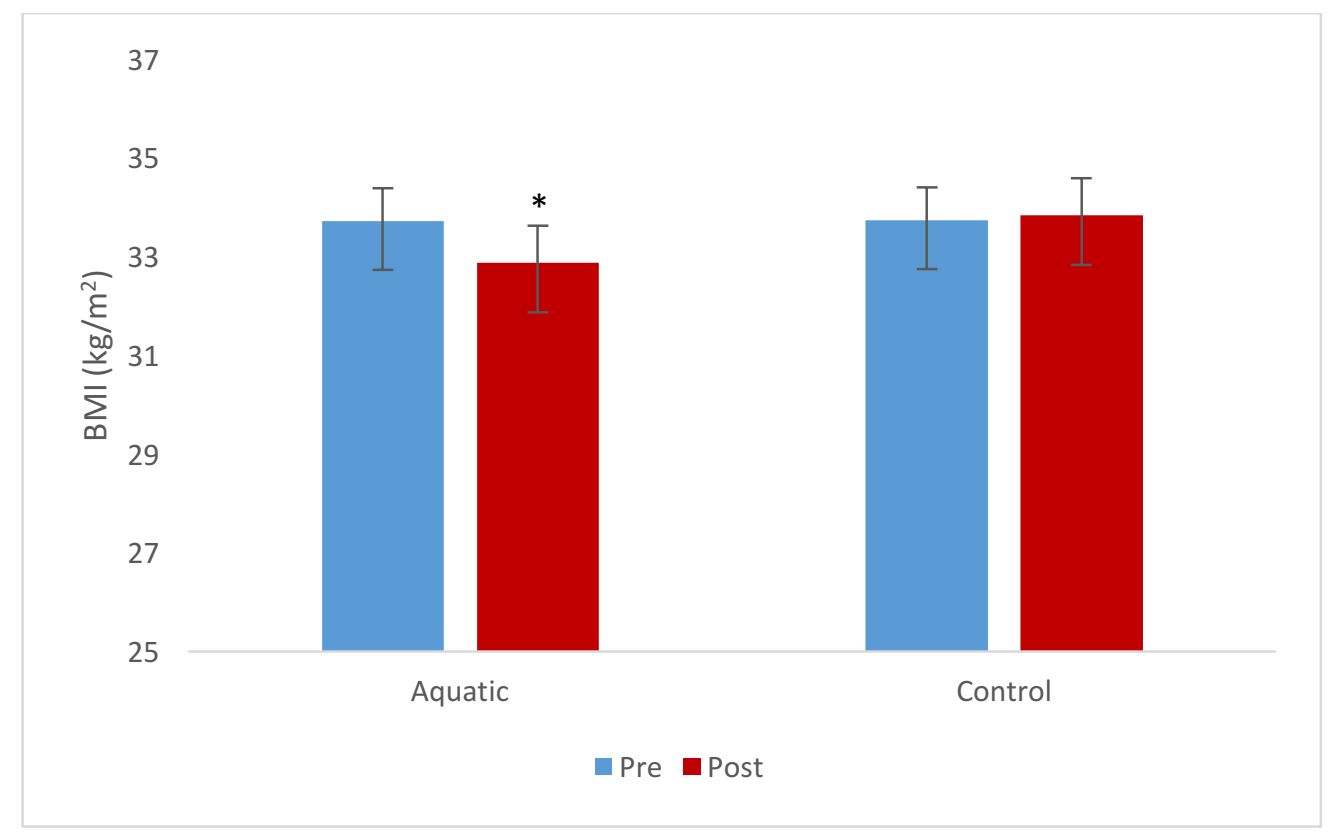

Figure 4.1: Changes in Body Mass Index (BMI) in Response to 8 weeks of Aquatic Exercise Compared to 8 Weeks of Non-Exercise Control. * Significant interaction from pre to post. The aquatic exercise group had a significant improvement in BMI when compared to the control, which showed no change.

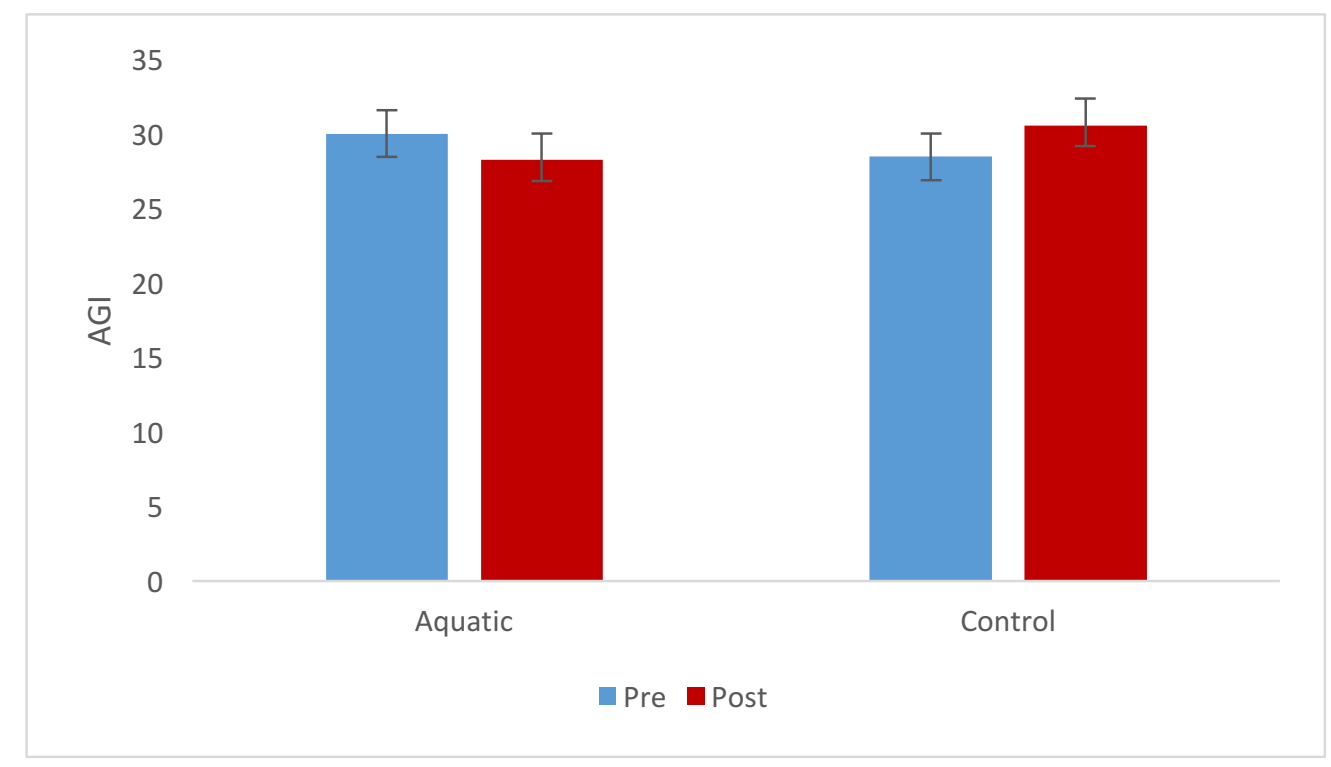

Figure 4.2: Changes in Augmentation Index (AGI) in Response to 8 Weeks of Aquatic Exercise Compared to 8

Weeks of Non-Exercise Control. There was no significant change in AGI over the course of the intervention. 


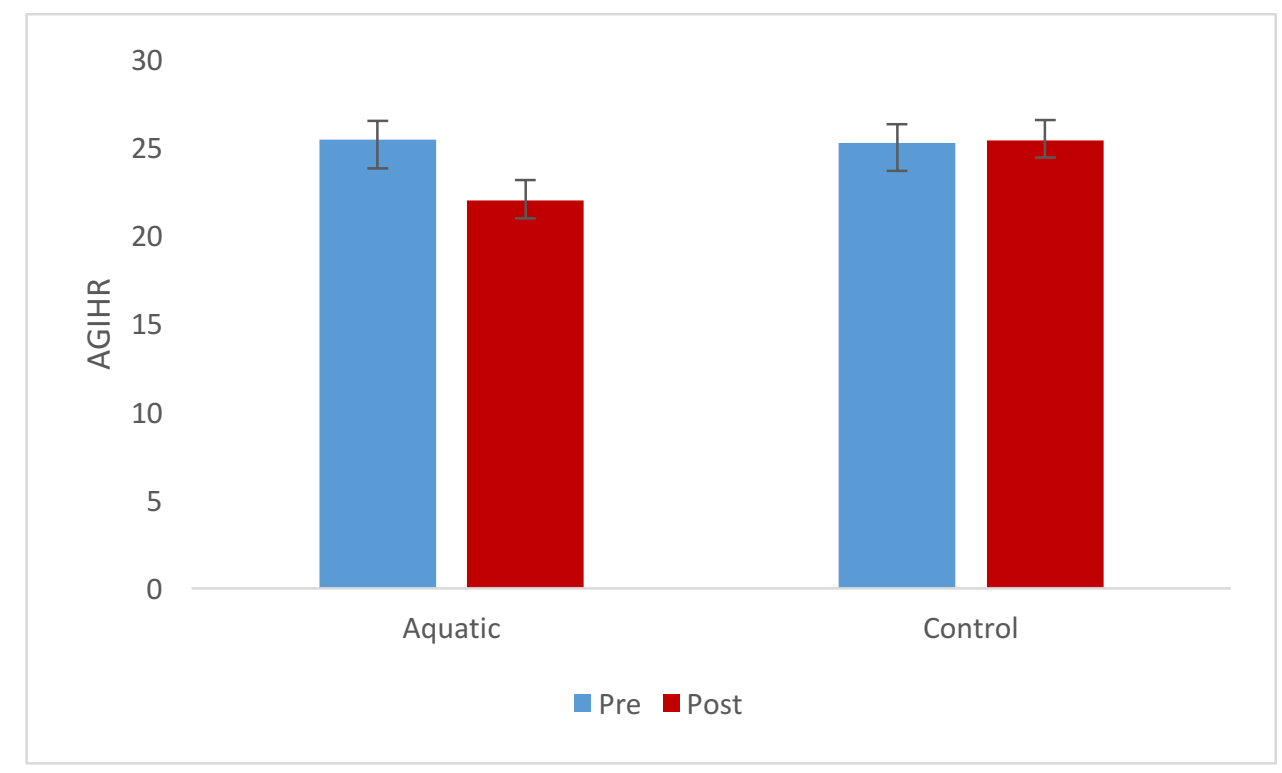

Figure 4.3: Changes in Augmentation Index, which has been Adjusted to a Standardized Heartrate (AGIHR) following 8 Weeks of Aquatic Exercise Compared to 8 Weeks of Non-Exercise Control. There was no significant change in AGIHR over the course of the intervention.

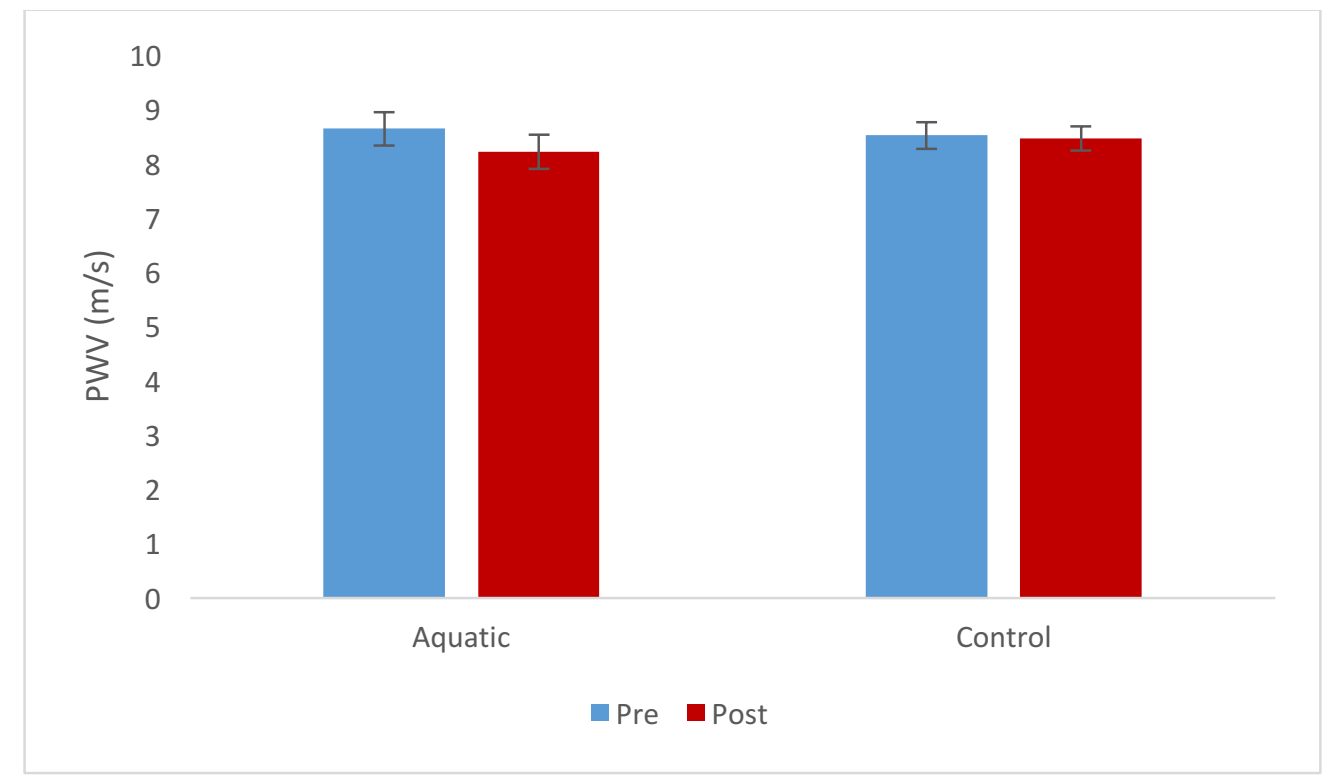

Figure 4.4: Changes in Pulse Wave Velocity (PWV) in Response to 8 Weeks of Aquatic Exercise Compared to 8 Weeks of Non-Exercise Control. There was no significant change in PWV over the course of the intervention. 
Table 4.1: Characteristics of the Study Cohort

\begin{tabular}{|c|c|c|c|c|}
\hline & \multicolumn{2}{|c|}{ AQUATIC EXERCISE } & \multicolumn{2}{|c|}{ CONTROL } \\
\hline & Pre & Post & Pre & Post \\
\hline HEIGHT (CM) & $165 \pm 2$ & - & $167 \pm 3$ & - \\
\hline WEIGHT (KG) & $91 \pm 4$ & $89 \pm 4$ & $95 \pm 7$ & $95 \pm 8$ \\
\hline SEX (\%FEMALE) & 100 & - & 100 & - \\
\hline $\mathrm{BSA}\left(\mathrm{M}^{2}\right)$ & $1.98 \pm 0.04$ & $1.95 \pm 0.04$ & $2.02 \pm 0.09$ & $2.01 \pm 0.09$ \\
\hline HIP CIRCUMFERENCE (CM) & $118 \pm 3$ & $115 \pm 4$ & $119 \pm 5$ & $115 \pm 5$ \\
\hline WAIST CIRCUMFERENCE (CM) & $101+3$ & $96 \pm 3$ & $105 \pm 4$ & $102 \pm 5$ \\
\hline BODY FAT (\%) & $44 \pm 2$ & $41 \pm 3$ & $40 \pm 2$ & $40 \pm 3$ \\
\hline
\end{tabular}

Values are means \pm SE. BSA, body surface area.

Table 4.2: Changes in Arterial Parameters Following 8 Weeks of Aquatic Exercise

Aquatic Exercise

Control

\begin{tabular}{|l|c|c|c|c|}
\multicolumn{2}{c}{} \\
\multicolumn{1}{l}{ Pre } & $62 \pm 4$ & $68 \pm 3$ & $64 \pm 3$ \\
\hline HR (BPM) & $66 \pm 4$ & $124 \pm 4$ & $128 \pm 4$ & $127 \pm 4$ \\
\hline bSBP (mmHg) & $129 \pm 5$ & $68 \pm 3$ & $76 \pm 5$ & $76 \pm 4$ \\
\hline bDBP (mmHg) & $73 \pm 3$ & $56 \pm 5$ & $53 \pm 5$ & $52 \pm 4$ \\
\hline bPP (mmHg) & $57 \pm 4$ & $88 \pm 2$ & $95 \pm 4$ & $95 \pm 4$ \\
\hline bMAP (mmHg) & $93 \pm 3$ & $115 \pm 5$ & $73 \pm 3$ & $69 \pm 3$ \\
\hline cSBP (mmHg) & $121 \pm 5$ & $69 \pm 3$ & $77 \pm 5$ & $76 \pm 4$ \\
\hline cDBP (mmHg) & $73 \pm 3$ & $46 \pm 6$ & $42 \pm 4$ & $42 \pm 3$ \\
\hline cPP (mmHg) & $47 \pm 5$ & $14 \pm 3$ & $12 \pm 2$ & $13 \pm 2$ \\
\hline AP (mmHg) & $15 \pm 3$ & $142 \pm 10$ & $138 \pm 9$ & $143 \pm 9$ \\
\hline SEVR (\%) & $134 \pm 8$ & & & \\
\hline
\end{tabular}

Values are Means+SE. AP, Augmentation Pressure; HR, heart rate; bSBP, brachial systolic blood pressure; bDBP, brachial diastolic blood pressure; cPP, central pulse pressure; CSBP, central systolic blood pressure; CDBP, central diastolic blood pressure; bMAP, brachial mean arterial pressure; SEVR, subendocardial variability ratio. 
Table 4.3: Changes in Blood Markers

\begin{tabular}{|l|c|c|c|c|}
\hline & Aquatic Exercise & \multicolumn{2}{c|}{ Pontrol } \\
\hline & Pre & Post & Pre \\
\hline Glucose (mg/dL) & $110 \pm 7$ & $101 \pm 7$ & $100 \pm 2$ & $98 \pm 2$ \\
\hline Insulin (mg/dL) & $19 \pm 2$ & $14 \pm 1$ & $16 \pm 3$ & $12 \pm 2$ \\
\hline HbA1c (mg/dL) & $6 \pm 0.3$ & $6 \pm 0.3$ & $6 \pm 0.1$ & $6 \pm 0.1$ \\
\hline Triglycerides (mg/dL) & $125 \pm 18$ & $141 \pm 17$ & $134 \pm 21$ & $146 \pm 12$ \\
\hline HDL (mg/dL) & $48 \pm 3$ & $48 \pm 3$ & $43 \pm 4$ & $42 \pm 4$ \\
\hline Total Cholesterol (mg/dL) & & & & $185 \pm 14$ \\
\hline
\end{tabular}

Values are Means+SE. HbA1c, glycated hemoglobin; HDL, high density lipoprotein.

Table 4.4: Changes in Exercise Capacity

AQUATIC EXERCISE

\begin{tabular}{|l|cccc|}
\hline & Pre & Post & Pre & Post \\
VO2 (ML/MIN/KG) & $15 \pm 1$ & $17 \pm 2$ & $14 \pm 1$ & $15 \pm 1$ \\
VCO2 (ML/MIN/KG) & $1 \pm 0.1$ & $2 \pm 0.2$ & $1 \pm 0.2$ & $1 \pm 0.2$ \\
RER & $1 \pm 0.01$ & $1 \pm 0.01$ & $1 \pm 0.02$ & $1 \pm 0.01$ \\
VE (L/MIN) & $54 \pm 6$ & $58 \pm 8$ & $50 \pm 5$ & $54 \pm 6$ \\
VT (L/MIN) & $1 \pm 0.1$ & $1 \pm 0.1$ & $1 \pm 0.1$ & $1 \pm 0.2$ \\
\hline
\end{tabular}

Values are Means+SE. VO2, Volume of oxygen consumption; VCO2, volume of expired carbon dioxide; RER, respiratory exchange ratio; VE, ventilatory equivalent; VT, Ventilatory threshold. 


\section{Chapter 5: General \\ Discussion}


General Discussion:

The purpose of this thesis was to investigate the efficacy of different modalities for offsetting AS in different age and health groups. More specifically, the goals were: 1) to assess the efficacy of aquatic exercise intervention on parameters of arterial stiffness, in a population of women with MetS; 2) to evaluate the efficacy of a fruit and vegetable based dietary intervention on parameters of AS, in a young population, free from overt CVD; 3) Determine if low carbohydrate or low fat diets work in coordination with fruit and vegetable dietary intervention to elicit changes in AS. Generally, our central hypothesis was that intervention, whether aquatic exercise or fruit and vegetable dietary intervention, would result in improvements in measures of AS. We also hypothesized that intervention in an older population with MetS would result in improvements in the risk factors associated with the development of MetS and CVD. Finally, we hypothesized that dietary intervention in a young population, would lead to improvements in the risk factors that predispose individuals for the development of MetS.

MetS is a cluster of comorbidities that lead to a host of complications, which ultimately result in an increased risk of CV mortality. This increased risk of mortality is characterized by a 3-fold increase in the risk for $\mathrm{CV}$ incident, such as myocardial infarction or cerebrovascular accident. Given that the current estimates show $35 \%$ of all adults and $50 \%$ of all adults over the age of 60 have MetS [80], working to develop an effective method to offset the negative effects associated with MetS, such as AS, is pertinent.

Aerobic exercise has been shown an effective method to offset the AS in those with MetS [1]. However, land based exercise compliance has traditionally been poor, with approximately $54 \%$ of adults in the US not meeting the federal guidelines for aerobic 
exercise and $33.9 \%$ of adults engaged in no aerobic exercise [96]. Therefore, an exercise modality effective at reducing AS and lowering participant dropout is necessary. The solution to this problem could potentially be aquatic base exercise. A major problem associated with land based exercise is the pain associated with it, particularly on the joints. This situation is alleviated in the aquatic environment, due to the buoyant effect of water, which decreases the load on the joints, and has been shown to decreased joint pain during and following bouts of exercise [97]. In addition, shallow water exercise has been shown to improve PWV following 10 weeks of intervention in a population of older individuals with CV risk factors [6]. The results of our aquatic intervention, as outlined in chapter 4, showed no significant improvements in any of the measures of AS. Therefore, at this time, our data does not support the notion that 8 weeks of aquatic exercise improves AS. Interestingly, even an intense exercise protocol, which increased from 45\%$75 \%$ HRR over the course of the study, we did not elicit a significant improvement in VO2 max. Given that there were no improvements in overall exercise capacity, perhaps the lack of improvements in AS, were associated with these lack of improvements in VO2 $\max$.

While it is of important to determine effective methods to offset AS in a population that has already diagnosed with MetS, it is also of great importance, perhaps more so, to determine an effective method to diminish the risk factors for the development of MetS in a young population. It has been postulated that treating hypertension at a younger age leads to better outcomes than treating it older in life, perhaps due to irreversible effects that occur with long-term uncontrolled hypertension [98]. Therefore, we desired to investigate treatment in a younger population, and we chose to utilize a dietary 
intervention, with increased fruit and vegetable consumption. While, we found no significant improvements in measures of AS, we did achieve significant improvements in bSBP, cSBP, and Buckberg SEVR, a measure of perfusion of the heart with blood. Reduction of cSBP by $2 \mathrm{mmHg}$ is associated with significantly reduced risk of vascular related mortality [76]. AS has been shown to precede and possibly contribute to the development of hypertension, therefore, these reductions in bSBP and cSBP are promising and may be protective for this younger population. It is of note, in the population we assessed, the PWV measurements were, on average, normal and moderately low, therefore, there was little room for improvement. This further supports the idea that the reduction in blood pressure may exert a protective effect on this population as they age [76].

Possible Mechanism for Arterial Improvements

While showing improvements in arterial parameters is of importance, it is of greater significance to elucidate the mechanism of improvement, in order to explain how and to develop more effective methods.

When considering improvements following dietary intervention we found significant decreases in the consumption of empty calories, which are often pre-packaged and processed foods, these often contain very high levels of sodium [99]. Sodium intake has been associated with hypertension, and a modest reduction in sodium intake has been shown to be an effective method for attenuating blood pressure [100]. There have been many theories as to what the mechanism behind these elevations in BP, with relation to sodium. It is postulated that elevated sodium ingestion leads to increased blood volume, 
which leads to an increased stroke volume due to the Starling mechanism [101]. Following this increase in stroke volume and consequently blood pressure, there is an increase in total peripheral resistance, which also supplements the increase in blood pressure [101]. Therefore, given the decrease in empty calories, we may have had a reduction in sodium consumption, which resulted or contributed to the decrease in the central and brachial blood pressures.

Measurements of cSBP are reflective of the blood pressure in the large conduit vessels, notably the aorta. Lowering cSBP has been associated with improvements in AS, due to the innate relationship between BP and AS [76]. One of the major components of the development of AS is structural stiffness, which is heavily influenced by BP [102]. An elevated BP often leads to a decrease in elastin fibers, an increase in collagen fibers, as well as an increase in collagen fiber crosslinks, these all lead to increased AS. Given that we showed these decreases in CSBP, following fruit and vegetable dietary intervention, it would be expected to see changes in measures of AS, given the intricate association between AS and central blood pressure. Our results are contrary to previous work, which show a direct relationship between changes in blood pressure and AS [103]. It is of note that this intervention was over the course of 8 weeks and showed no change in measures of AS, thus it is possible that the intervention require a longer intervention period to elicit changes in AS.

Sympathetic nervous system (SNS) stimulation has been shown to have an effect of the arteries, which has been proven in multiple studies evaluating the blockage or removal of SNS stimulation $[104,105]$. It has been suggested that insulin resistance, a component of MetS, leads to an increased activation of the SNS [28]. This increase in 
SNS activation leads to reduced arterial distensibility, which in turns leads to elevated stiffness, due to the increased tone of the arterial smooth muscle [106]. Additionally, SNS activation may lead to contraction of vascular smooth muscle cells (VSMC), which leads to greater tone of the artery, and thus increased stiffness [107].

NO is a potent vasodilator and is one of the major modulators of arterial tone, through interaction with the endothelium of the arteries [30]. Therefore, with improvements in the bioavailability of NO or better handling of NO by the endothelium, there will also be reductions in AS [79]. These factors are both blunted in a population with MetS [79]. It has also been shown previously that land based exercise can lead to improvements in endothelial function, the mechanism through which these improvements in endothelial function occur is likely due to the increased shear stress that is exerted on the arterial walls while exercising [108]. Shear stress is the force that is exerted on the walls of the artery as the blood is pumped, and increases in the environment of exercise. Exercise induces a state of hyperemia, where there is strain, shear stress, placed on the walls of the artery. This shear stress over time can lead to improvements in the vessel's ability to dilate and modulate tone in order to accommodate the increased strain. This occurs through a mechanism, in which endothelial nitric oxide synthase (eNOS) is activated [109]. 


\section{Future Directions:}

The findings presented in this thesis are promising and show the efficacy of fruit and vegetable dietary intervention at improving both central and brachial blood pressure. These findings help to build the foundation on which we can build to further elucidate mechanism and determine better methods to assess $\mathrm{CV}$ improvements following nutrition or exercise intervention.

Our findings suggest that neither aquatic exercise nor nutritional intervention was effective at the reduction of AS in our population. Given these findings, it may be of interest to examine a combined nutrition and exercise intervention. There is limited literature available examining a dietary intervention that is used in conjunction with exercise. Also of interest, investigating the use of particular food sources in conjunction with exercise, to determine if there is a specific component of food that may lead to improvements in AS. Beet root juice has previously been shown to transiently reduce PWV following 1 acute dose [41], this is speculated to be due to the nitrate and nitrite present. The nitrate and nitrite are converted into NO, which leads to increased vasodilation, however, the effects of chronic supplementation of beet root juice on AS, has not been investigated as heavily [110].

The SNS is a major modulator of arterial tone, through the baroreflex. The baroreflex is the body's method of coping with changes in blood pressure, by changes in the tone of the arteries [111]. The baroreflex sensitivity (BRS) can be measured, by detecting changes in the DBP following each contraction of the heart. It is speculated that there is a relationship between AS and the BRS, and both can be improved with aerobic exercise training in both an animal model and in a diseased human model [112]. 
Therefore, it may be of interest to determine if the improvements that we saw in AS were related to baroreflex sensitivity.

Mechanistically, changes in AS can be detected by biomarkers in the blood, such as matrix metalloproteinase-9 (MMP-9), MMP-2, C-reactive protein (CRP), and interleukin-6 (IL-6) $[27,113]$. MMP-9 and MMP-2 are both enzymes that are created in the body and are involved in remodeling of the arterial walls. MMP-9 and MMP-2 are associated with increased arterial stiffness, due to the breakdown of elastin [27]. Inflammatory states have been associated with AS particularly CRP and IL-6. This association is speculated to be caused by alterations to arterial wall distensibility [113].

Our nutritional intervention was implemented in a college age population, which tend to have lower measures of AS likely explaining our results. These lower measures of AS, meaning that there is limited room for improvement. It would be of interest to implement this intervention in a population with elevated AS, such as those with MetS. It would be reasonable to postulate that there may be reductions in AS in this unhealthy population.

Overall, our data provide many initial findings, which on a larger scale may be able to produce more significant results. Given that we have shown that aquatic exercise shows a trend toward improvement in measures of AS, increasing the sample size would be important to determine if the trend toward improvement become statistically significant. If indeed they do show significance, it is of importance to elucidate the mechanism through which the improvements in AS are occurring. Therefore, follow up studies with additional measures, such as endothelial function, BRS, blood biomarkers (MMP, CRP, 
and $T N F \alpha$ ), would help to determine the mechanism. While in the nutritional intervention, would be of interest to determine whether it would be effective in diseased population. 


\section{Limitations:}

All studies are with limitations and those presented in this thesis are of no exception. The objective of the two studies was to investigate whether an intervention, either a diet with increased fruits and vegetables or aquatic exercise, would lead to changes in AS.

Similar to most nutrition based research, this study could allow for a self-report bias. In order to combat this, all subjects were educated on the necessity that all data be reported accurately, we also asked them to give all of their receipts, and we asked the participants to take pictures of their meals. Another potential limitation is that the results could be confounded by other potential mechanisms. It is of note; the participants were asked to maintain their current physical activity level. We assessed their movement with an accelerometer at baseline and the final week of the study to determine if there were any major differences in their movement. Unfortunately, the subjects were not measured for the entire 8 weeks of the intervention, so it is possible that the duration the participants had a change in exercise, which were not detected. Finally, the diet was not restrictive in terms of what fruits and vegetables could be consumed, therefore, the subjects could have eaten many vasoactive fruits and vegetables or none. This could have impacted our results, in terms of who improved AS and those who didn't.

The largest limiting factor, which may have affected the aquatic exercise results, is the small sample size. A sample size as small as this one is not able to show statistical significance in the measures that were taken. Many of our measurements show a trend toward improvement and we believe that the results of this study will be 
more significant with the recruitment of more participants. Participants were particularly difficult to recruit for this study due to the limited availability of the aquatic exercise classes, which were only available twice a day, but both were during the working day. Another major limitation is that $100 \%$ of the participants that we recruited were women, therefore, we cannot extrapolate our results to men as well. We also did not ask the participants to keep a log of their dietary intake, which could lead to confounding factors, where their diet could have been related to some of their improvements. 


\section{References:}

1. Donley, D.A., et al., Aerobic exercise training reduces arterial stiffness in metabolic syndrome. J Appl Physiol (1985), 2014. 116(11): p. 1396-404.

2. Macready, A.L., et al., Flavonoid-rich fruit and vegetables improve microvascular reactivity and inflammatory status in men at risk of cardiovascular disease--FLAVURS: a randomized controlled trial. Am J Clin Nutr, 2014. 99(3): p. 479-89.

3. Berrones, B.S.F.a.A.J., Arterial Stiffness Implications and Interventions. Springer Briefs in Physiology. 2015, Switzerland: Springer. 61.

4. $\quad$ Farahani, A.V., et al., The effects of a 10-week water aerobic exercise on the resting blood pressure in patients with essential hypertension. Asian J Sports Med, 2010. 1(3): p. 159-67.

5. Tanaka, H., et al., Swimming training lowers the resting blood pressure in individuals with hypertension. J Hypertens, 1997. 15(6): p. 651-7.

6. Sherlock, L., et al., Effects of Shallow Water Aerobic Exercise Training on Arterial Stiffness and Pulse Wave Analysis in Older Individuals. International Journal of Aquatic Research \& Education, 2014. 8(4): p. 310-320.

7. George, T.W., et al., Effects of chronic consumption of fruit and vegetable puree-based drinks on vasodilation, plasma oxidative stability and antioxidant status. J Hum Nutr Diet, 2012. 25(5): p. 477-87.

8. George, T.W., et al., Effects of acute consumption of a fruit and vegetable puree-based drink on vasodilation and oxidative status. Br J Nutr, 2013. 109(8): p. 1442-52.

9. He, F.J., et al., Increased consumption of fruit and vegetables is related to a reduced risk of coronary heart disease: meta-analysis of cohort studies. J Hum Hypertens, 2007. 21(9): p. 717-28.

10. McCall, D.O., et al., Dietary intake of fruits and vegetables improves microvascular function in hypertensive subjects in a dose-dependent manner. Circulation, 2009. 119(16): p. 2153-60.

11. Oude Griep, L.M., et al., Raw and processed fruit and vegetable consumption and 10year coronary heart disease incidence in a population-based cohort study in the Netherlands. PLoS One, 2010. 5(10): p. e13609.

12. Aatola, H., et al., Lifetime fruit and vegetable consumption and arterial pulse wave velocity in adulthood: the Cardiovascular Risk in Young Finns Study. Circulation, 2010. 122(24): p. 2521-8.

13. Ellingsen, I., et al., Vitamin C consumption is associated with less progression in carotid intima media thickness in elderly men: A 3-year intervention study. Nutr Metab Cardiovasc Dis, 2009. 19(1): p. 8-14.

14. Ellingsen, I., et al., Consumption of fruit and berries is inversely associated with carotid atherosclerosis in elderly men. Br J Nutr, 2008. 99(3): p. 674-81.

15. Monahan, K.D., et al., Effect of omega-3 polyunsaturated fatty acid supplementation on central arterial stiffness and arterial wave reflections in young and older healthy adults. Physiol Rep, 2015. 3(6).

16. Mozaffarian, D., L.J. Appel, and L. Van Horn, Components of a cardioprotective diet: new insights. Circulation, 2011. 123(24): p. 2870-91. 
17. Gomez-Delgado, F., et al., Chronic consumption of a low-fat diet improves cardiometabolic risk factors according to the CLOCK gene in patients with coronary heart disease. Mol Nutr Food Res, 2015. 59(12): p. 2556-64.

18. Wang, X., et al., Short-term intensive atorvastatin therapy improves endothelial function partly via attenuating perivascular adipose tissue inflammation through 5lipoxygenase pathway in hyperlipidemic rabbits. Chin Med J (Engl), 2014. 127(16): p. 2953-9.

19. Falcone Ferreyra, M.L., S.P. Rius, and P. Casati, Flavonoids: biosynthesis, biological functions, and biotechnological applications. Front Plant Sci, 2012. 3: p. 222.

20. Kochanek, K.D., et al., Mortality in the United States, 2013. NCHS Data Brief, 2014(178): p. 1-8.

21. Finkelstein, E.A., et al., Annual medical spending attributable to obesity: payer-and service-specific estimates. Health Aff (Millwood), 2009. 28(5): p. w822-31.

22. Ogden, C.L. and National Center for Health Statistics (U.S.), Prevalence of obesity among adults : United States, 2011-2012. NCHS data brief. 1 online resource (7 pages).

23. Le, J., et al., "Vascular age" is advanced in children with atherosclerosis-promoting risk factors. Circ Cardiovasc Imaging, 2010. 3(1): p. 8-14.

24. Reaven, G.M., Banting lecture 1988. Role of insulin resistance in human disease. Diabetes, 1988. 37(12): p. 1595-607.

25. Grundy, S.M., et al., Definition of metabolic syndrome: report of the National Heart, Lung, and Blood Institute/American Heart Association conference on scientific issues related to definition. Arterioscler Thromb Vasc Biol, 2004. 24(2): p. e13-8.

26. Zieman, S.J., V. Melenovsky, and D.A. Kass, Mechanisms, pathophysiology, and therapy of arterial stiffness. Arterioscler Thromb Vasc Biol, 2005. 25(5): p. 932-43.

27. Yasmin, et al., Matrix metalloproteinase-9 (MMP-9), MMP-2, and serum elastase activity are associated with systolic hypertension and arterial stiffness. Arterioscler Thromb Vasc Biol, 2005. 25(2): p. 372.

28. Chantler, P.D. and J.C. Frisbee, Arterial function in cardio-metabolic diseases: from the microcirculation to the large conduits. Prog Cardiovasc Dis, 2015. 57(5): p. 489-96.

29. Fleming, I. and R. Busse, Signal transduction of eNOS activation. Cardiovasc Res, 1999. 43(3): p. 532-41.

30. Pober, J.S., W. Min, and J.R. Bradley, Mechanisms of endothelial dysfunction, injury, and death. Annu Rev Pathol, 2009. 4: p. 71-95.

31. Chan, H.T., et al., Worsened arterial stiffness in high-risk cardiovascular patients with high habitual carbohydrate intake: a cross-sectional vascular function study. BMC Cardiovasc Disord, 2014. 14: p. 24.

32. Santana, A.B., et al., Effect of high-fat diet upon inflammatory markers and aortic stiffening in mice. Biomed Res Int, 2014. 2014: p. 914102.

33. Safar, M.E., et al., Sodium intake and vascular stiffness in hypertension. Hypertension, 2009. 54(2): p. 203-9.

34. Farquhar, W.B., et al., Dietary sodium and health: more than just blood pressure. J Am Coll Cardiol, 2015. 65(10): p. 1042-50.

35. Oberleithner, H., et al., Salt overload damages the glycocalyx sodium barrier of vascular endothelium. Pflugers Arch, 2011. 462(4): p. 519-28. 
36. Ellins, E., et al., Arterial stiffness and inflammatory response to psychophysiological stress. Brain Behav Immun, 2008. 22(6): p. 941-8.

37. Jovanovski, E., A. Zurbau, and V. Vuksan, Carbohydrates and endothelial function: is a low-carbohydrate diet or a low-glycemic index diet favourable for vascular health? Clin Nutr Res, 2015. 4(2): p. 69-75.

38. Bondonno, C.P., et al., Short-term effects of nitrate-rich green leafy vegetables on blood pressure and arterial stiffness in individuals with high-normal blood pressure. Free Radic Biol Med, 2014. 77: p. 353-62.

39. Lynn, A., et al., Effects of pomegranate juice supplementation on pulse wave velocity and blood pressure in healthy young and middle-aged men and women. Plant Foods Hum Nutr, 2012. 67(3): p. 309-14.

40. McAnulty, L.S., et al., Six weeks daily ingestion of whole blueberry powder increases natural killer cell counts and reduces arterial stiffness in sedentary males and females. Nutr Res, 2014. 34(7): p. 577-84.

41. Kim, J.K., et al., Acute dietary nitrate supplementation does not augment submaximal forearm exercise hyperemia in healthy young men. Appl Physiol Nutr Metab, 2015. 40(2): p. 122-8.

42. Hwang, M.H., et al., Validity and reliability of aortic pulse wave velocity and augmentation index determined by the new cuff-based SphygmoCor Xcel. J Hum Hypertens, 2014. 28(8): p. 475-81.

43. McClintock, T.R., et al., Major dietary patterns and carotid intima-media thickness in Bangladesh. Public Health Nutr, 2015: p. 1-12.

44. Madisetty, M.K., et al., Assessment of Oxidative Stress Markers and Carotid Artery Intima-Media Thickness in Elderly Patients Without and with Coronary Artery Disease. Indian J Clin Biochem, 2016. 31(3): p. 278-85.

45. Petersen, K.S., et al., Effect of improving dietary quality on carotid intima media thickness in subjects with type 1 and type 2 diabetes: a 12-mo randomized controlled trial. Am J Clin Nutr, 2015. 102(4): p. 771-9.

46. Svedenhag, J. and J. Seger, Running on land and in water: comparative exercise physiology. Med Sci Sports Exerc, 1992. 24(10): p. 1155-60.

47. Nualnim, N., et al., Effects of swimming training on blood pressure and vascular function in adults $>50$ years of age. Am J Cardiol, 2012. 109(7): p. 1005-10.

48. Lee, B.A. and D.J. Oh, The effects of aquatic exercise on body composition, physical fitness, and vascular compliance of obese elementary students. J Exerc Rehabil, 2014. 10(3): p. 184-90.

49. Alkatan, M., et al., Effects of Swimming and Cycling Exercise Intervention on Vascular Function in Patients With Osteoarthritis. Am J Cardiol, 2016. 117(1): p. 141-5.

50. Arca, E.A., et al., Aquatic exercise is as effective as dry land training to blood pressure reduction in postmenopausal hypertensive women. Physiother Res Int, 2014. 19(2): p. 93-8.

51. Mohr, M., et al., High-intensity intermittent swimming improves cardiovascular health status for women with mild hypertension. Biomed Res Int, 2014. 2014: p. 728289.

52. Co, J., et al., Obesity, Hypertension and Metabolic Syndrome in Children in West Virginia. W V Med J, 2015. 111(4): p. 20-4.

53. Morrell, J.S., et al., Metabolic syndrome, obesity, and related risk factors among college men and women. J Am Coll Health, 2012. 60(1): p. 82-9. 
54. Bertel, 0., et al., Decreased Beta-adrenoreceptor responsiveness as related to age, blood pressure, and plasma catecholamines in patients with essential hypertension. Hypertension, 1980. 2(2): p. 130-8.

55. Mir, S., Y. Tabel, and S. Darcan, Is presence of hypertension in obese children correlate with the criteria of metabolic syndrome? J Trop Pediatr, 2007. 53(6): p. 424-7.

56. Nutrition, Physical Activity and Obesity Data, Trends and Maps web site. U.S. Department of Health and Human Services, Centers for Disease Control and Prevention (CDC), National Center for Chronic Disease Prevention and Health Promotion, Division of Nutrition, Physical Activity and Obesity, Atlanta, GA, 2015. Available at http://www.cdc.gov/nccdphp/DNPAO/index.html.

57. Tilton, R.G., et al., Vascular dysfunction induced by elevated glucose levels in rats is mediated by vascular endothelial growth factor. J Clin Invest, 1997. 99(9): p. 2192202.

58. Jablonski, K.L., et al., Dietary sodium restriction and association with urinary marinobufagenin, blood pressure, and aortic stiffness. Clin J Am Soc Nephrol, 2013. 8(11): p. 1952-9.

59. Lithander, F.E., et al., Postprandial effect of dietary fat quantity and quality on arterial stiffness and wave reflection: a randomised controlled trial. Nutr J, 2013. 12: p. 93.

60. Bailey, B.W., et al., Adherence to the 2010 Dietary Guidelines for Americans and the relationship to adiposity in young women. J Nutr Educ Behav, 2015. 47(1): p. 86-93.

61. Tanaka, H. and M.E. Safar, Influence of lifestyle modification on arterial stiffness and wave reflections. Am J Hypertens, 2005. 18(1): p. 137-44.

62. Ebbeling, C.B., et al., Effects of a low-glycemic load vs low-fat diet in obese young adults: a randomized trial. JAMA, 2007. 297(19): p. 2092-102.

63. Mirrahimi, A., et al., The role of glycemic index and glycemic load in cardiovascular disease and its risk factors: a review of the recent literature. Curr Atheroscler Rep, 2014. 16(1): p. 381.

64. Benjamin, R.M., Dietary guidelines for Americans, 2010: the cornerstone of nutrition policy. Public Health Rep, 2011. 126(3): p. 310-1.

65. Chen, C.H., et al., Estimation of central aortic pressure waveform by mathematical transformation of radial tonometry pressure. Validation of generalized transfer function. Circulation, 1997. 95(7): p. 1827-36.

66. Chen, C.H., et al., Validation of carotid artery tonometry as a means of estimating augmentation index of ascending aortic pressure. Hypertension, 1996. 27(2): p. 16875.

67. Salvi, P., et al., Changes in subendocardial viability ratio with acute high-altitude exposure and protective role of acetazolamide. Hypertension, 2013. 61(4): p. 793-9.

68. Butlin, M., et al., Carotid-femoral pulse wave velocity assessment using novel cuffbased techniques: comparison with tonometric measurement. J Hypertens, 2013. 31(11): p. 2237-43; discussion 2243.

69. Tabas, I., Cholesterol in health and disease. J Clin Invest, 2002. 110(5): p. 583-90.

70. Williamson, D.A., et al., Comparison of digital photography to weighed and visual estimation of portion sizes. J Am Diet Assoc, 2003. 103(9): p. 1139-45.

71. Camps, G., et al., Empty calories and phantom fullness: a randomized trial studying the relative effects of energy density and viscosity on gastric emptying determined by MRI and satiety. Am J Clin Nutr, 2016. 104(1): p. 73-80. 
72. Weisbrod, R.M., et al., Arterial stiffening precedes systolic hypertension in diet-induced obesity. Hypertension, 2013. 62(6): p. 1105-10.

73. Gupta, R. and S. Guptha, Strategies for initial management of hypertension. Indian J Med Res, 2010. 132: p. 531-42.

74. Daniels, S.R., et al., Overweight in children and adolescents: pathophysiology, consequences, prevention, and treatment. Circulation, 2005. 111(15): p. 1999-2012.

75. U.S. Department of Health and Human Services and U.S. Department of Agriculture. 2015 - 2020 Dietary Guidelines for Americans. 8th Edition. December 2015. Available at http://health.gov/dietaryguidelines/2015/guidelines/.

76. Lewington, S., et al., Age-specific relevance of usual blood pressure to vascular mortality: a meta-analysis of individual data for one million adults in 61 prospective studies. Lancet, 2002. 360(9349): p. 1903-13.

77. Bosse, J.D., et al., A low-carbohydrate/high-fat diet reduces blood pressure in spontaneously hypertensive rats without deleterious changes in insulin resistance. Am J Physiol Heart Circ Physiol, 2013. 304(12): p. H1733-42.

78. Roberts, C.K., et al., Enhanced NO inactivation and hypertension induced by a high-fat, refined-carbohydrate diet. Hypertension, 2000. 36(3): p. 423-9.

79. Endemann, D.H. and E.L. Schiffrin, Nitric oxide, oxidative excess, and vascular complications of diabetes mellitus. Curr Hypertens Rep, 2004. 6(2): p. 85-9.

80. Aguilar, M., et al., Prevalence of the metabolic syndrome in the United States, 20032012. JAMA, 2015. 313(19): p. 1973-4.

81. Malik, S., et al., Impact of the metabolic syndrome on mortality from coronary heart disease, cardiovascular disease, and all causes in United States adults. Circulation, 2004. 110(10): p. 1245-50.

82. Vlachopoulos, C., K. Aznaouridis, and C. Stefanadis, Prediction of cardiovascular events and all-cause mortality with arterial stiffness: a systematic review and metaanalysis. J Am Coll Cardiol, 2010. 55(13): p. 1318-27.

83. Booth, A.D., et al., Inflammation and arterial stiffness in systemic vasculitis: a model of vascular inflammation. Arthritis Rheum, 2004. 50(2): p. 581-8.

84. Hayashi, K., et al., Effects of aerobic exercise training on the stiffness of central and peripheral arteries in middle-aged sedentary men. Jpn J Physiol, 2005. 55(4): p. 2359.

85. Kearney, T.M., et al., Accumulated brisk walking reduces arterial stiffness in overweight adults: evidence from a randomized control trial. J Am Soc Hypertens, 2014. 8(2): p. 117-26.

86. Maeda, S., et al., Aortic stiffness and aerobic exercise: mechanistic insight from microarray analyses. Med Sci Sports Exerc, 2005. 37(10): p. 1710-6.

87. Nakanishi, Y., T. Kimura, and Y. Yokoo, Maximal physiological responses to deep water running at thermoneutral temperature. Appl Human Sci, 1999. 18(2): p. 31-5.

88. Barbagallo, M., et al., Altered ionic effects of insulin in hypertension: role of basal ion levels in determining cellular responsiveness. J Clin Endocrinol Metab, 1997. 82(6): p. 1761-5.

89. Resnick, L.M., et al., Direct magnetic resonance determination of aortic distensibility in essential hypertension: relation to age, abdominal visceral fat, and in situ intracellular free magnesium. Hypertension, 1997. 30(3 Pt 2): p. 654-9. 
90. Clarkson, P., et al., Exercise training enhances endothelial function in young men. J Am Coll Cardiol, 1999. 33(5): p. 1379-85.

91. Kasliwal, R.R., et al., Effect of pistachio nut consumption on endothelial function and arterial stiffness. Nutrition, 2015. 31(5): p. 678-85.

92. Jennings, A., et al., Higher anthocyanin intake is associated with lower arterial stiffness and central blood pressure in women. Am J Clin Nutr, 2012. 96(4): p. 781-8.

93. Bastard, J.P., et al., Adipose tissue IL-6 content correlates with resistance to insulin activation of glucose uptake both in vivo and in vitro. J Clin Endocrinol Metab, 2002. 87(5): p. 2084-9.

94. McLaughlin, T., et al., Differentiation between obesity and insulin resistance in the association with C-reactive protein. Circulation, 2002. 106(23): p. 2908-12.

95. Park, S. and E.G. Lakatta, Role of inflammation in the pathogenesis of arterial stiffness. Yonsei Med J, 2012. 53(2): p. 258-61.

96. Schoenborn, C.A., P.F. Adams, and J.A. Peregoy, Health behaviors of adults: United States, 2008-2010. Vital Health Stat 10, 2013(257): p. 1-184.

97. Wyatt, F.B., et al., The effects of aquatic and traditional exercise programs on persons with knee osteoarthritis. J Strength Cond Res, 2001. 15(3): p. 337-40.

98. Turnbull, F., et al., Effects of different regimens to lower blood pressure on major cardiovascular events in older and younger adults: meta-analysis of randomised trials. BMJ, 2008. 336(7653): p. 1121-3.

99. Lucan, S.C., A. Karpyn, and S. Sherman, Storing empty calories and chronic disease risk: snack-food products, nutritive content, and manufacturers in Philadelphia corner stores. J Urban Health, 2010. 87(3): p. 394-409.

100. Chrysant, S.G., Effects of High Salt Intake on Blood Pressure and Cardiovascular Disease: The Role of COX Inhibitors. Clin Cardiol, 2016. 39(4): p. 240-2.

101. Haddy, F.J., Mechanism, prevention and therapy of sodium-dependent hypertension. Am J Med, 1980. 69(5): p. 746-58.

102. Payne, R.A., I.B. Wilkinson, and D.J. Webb, Arterial stiffness and hypertension: emerging concepts. Hypertension, 2010. 55(1): p. 9-14.

103. Lim, J., et al., Impact of blood pressure perturbations on arterial stiffness. Am J Physiol Regul Integr Comp Physiol, 2015. 309(12): p. R1540-5.

104. Grassi, G., et al., Sympathetic modulation of radial artery compliance in congestive heart failure. Hypertension, 1995. 26(2): p. 348-54.

105. Kosch, M., et al., Relationship between muscle sympathetic nerve activity and large artery mechanical vessel wall properties in renal transplant patients. J Hypertens, 2002. 20(3): p. 501-8.

106. Lichtor, T., et al., The sympathetic nervous system and atherosclerosis. J Neurosurg, 1987. 67(6): p. 906-14.

107. Dobrin, P.B. and A.A. Rovick, Influence of vascular smooth muscle on contractile mechanics and elasticity of arteries. Am J Physiol, 1969. 217(6): p. 1644-51.

108. Higashi, Y., et al., Regular aerobic exercise augments endothelium-dependent vascular relaxation in normotensive as well as hypertensive subjects: role of endotheliumderived nitric oxide. Circulation, 1999. 100(11): p. 1194-202.

109. Davis, M.E., et al., Shear stress regulates endothelial nitric-oxide synthase promoter activity through nuclear factor kappaB binding. J Biol Chem, 2004. 279(1): p. 163-8. 
110. Paniagua, O.A., M.B. Bryant, and J.A. Panza, Role of endothelial nitric oxide in shear stress-induced vasodilation of human microvasculature: diminished activity in hypertensive and hypercholesterolemic patients. Circulation, 2001. 103(13): p. 17528.

111. Michas, F., et al., Baroreceptor reflex sensitivity is associated with arterial stiffness in a population of normotensive and hypertensive patients. Blood Press Monit, 2012. 17(4): p. 155-9.

112. Irigoyen, M.C., et al., Exercise training improves baroreflex sensitivity associated with oxidative stress reduction in ovariectomized rats. Hypertension, 2005. 46(4): p. 9981003.

113. Schnabel, R., et al., Relations of inflammatory biomarkers and common genetic variants with arterial stiffness and wave reflection. Hypertension, 2008. 51(6): p. 1651-7. 